\title{
Organic/polymer photothermal nanoagents for photoacoustic imaging and photothermal therapy in vivo
}

\author{
Hanlin Ou ${ }^{1}$, Jun $\mathrm{Li}^{1}$, Chao Chen ${ }^{1}$, Heqi Gao ${ }^{1}, \mathrm{Xue}_{\mathrm{Xue}}{ }^{2^{*}}$ and Dan Ding ${ }^{1,2,3^{*}}$
}

\begin{abstract}
In recent years, organic/polymer photothermal nanoagents including semiconducting polymer nanoparticles and small-molecule organic photothermal agents-encapsulated nanoparticles have attracted large attention from researchers in the biomedical field, owing to their excellent optical properties, good biocompatibility, easy processability, and flexible surface functionalization, as well as their combined functions of photoacoustic (PA) imaging and photothermal therapy (PTT). In this review, we summarize the recent advances in organic/ polymer photothermal nanoagents for in vivo PA imaging and PTT applications. In particular, we focus on the design strategies, which are composed of traditional approaches and emerging mechanisms, especially based on "intramolecular motion-induced photothermy" strategy to regulate the photophysical properties of organic/polymer photothermal nanoagents for boosted in vivo PA imaging and PTT.
\end{abstract}

Keywords: photothermal agent, photoacoustic imaging, photothermal therapy, semiconducting polymer nanoparticle

\section{INTRODUCTION}

Recently, photothermal agents with the combined functions of photoacoustic (PA) imaging and photothermal therapy (PTT) have aroused large attention in the biomedical field, as they permit real-time diagnosis and concurrent in situ phototherapy [1-7]. Upon short-pulsed laser irradiation, photothermal agents absorb the energy of the laser light and partially convert it into heat, resulting in local instantaneous thermal expansion and subsequent ultrasonic emission [8-10]. By collecting these photothermally generated ultrasound signals through a broadband ultrasonic transducer and transforming them into images, PA imaging integrates the merits of high contrast of optical imaging with high spatial resolution of ultrasound imaging, realizing disease diagnosis in deeper tissue with high contrast and resolution [11-13]. Owing to their excellent heat generation ability, photothermal agents are also widely used in PTT [4,14-17]. Attractively, the two functions of photothermal agents, PA imaging and PTT, are complementary [18]. PA imaging could guide PTT, e.g., in the diagnosis and treatment of tumors, PA imaging enables the diagnosis of tumor location and the monitoring of PTT effect in real time [19-22]. While the strong PTT inhibits the tumor growth effectively accompanied with a high PA signal $[23,24]$.

The rise of nanotechnology since the 1990s has advanced the field of cancer theranostics. Nanomaterials, in particular the nanoparticles (NPs) have shown unique merits such as improved water-solubility of payload, prolonged blood circulation time and enhanced permeability and retention (EPR) effect [3,25-27]. To date, a variety of photothermal nanoagents for PA imaging and PTT have been reported, including NPs based on noble metal materials and transition metal dichalcogenides, carbon nanomaterials and organic/polymer-based NPs [28-41]. While these photothermal nanoagents have their own merits, each has a drawback. For instance, noble metal materials and transition metal dichalcogenides suffer from long-term toxicity issues in terms of clinical application, and carbon nanomaterials are restricted by their flat PA spectra $[42,43]$. Among these photothermal nanoagents, organic/polymer-based NPs have gained

\footnotetext{
${ }^{1}$ Key Laboratory of Bioactive Materials, Ministry of Education, and College of Life Sciences, Nankai University, Tianjin 300071, China

${ }^{2}$ State Key Laboratory of Medicinal Chemical Biology, and College of Pharmacy, Nankai University, Tianjin 300353, China

${ }^{3}$ Jiangsu Center for the Collaboration and Innovation of Cancer Biotherapy, Cancer Institute, Xuzhou Medical University, Xuzhou 221002, China

* Corresponding authors (emails: dingd@nankai.edu.cn (Ding D); xuexue@nankai.edu.cn (Xue X))
} 
extensive interest due to their excellent optical properties, good biocompatibility, easy processability, and flexible surface functionalization $[44,45]$. The PA and PTT efficiencies of the organic/polymer photothermal nanoagents are significantly affected by their extinction coefficients, non-radiative decay efficiencies and Grüneisen coefficients [33]. By improving any of these above parameters, massive researches have been proven to improve the PA imaging and PTT effect [34-39]. Organic/polymer photothermal nanoagents utilized in PA imaging and PTT could be roughly divided into two main groups: semiconducting polymer NPs (SPNs) and small-molecule organic photothermal agents-encapsulated NPs [46,47].

So far, although there have been many review articles summarizing the development of photothermal agents for PA imaging and PTT $[2,14,23,48]$, rather limited ones focus on the molecular/NP design and recently developed new mechanisms of the organic/polymer photothermal nanoagents. In this review, we summarize the recent advances in the design strategies of organic/polymer photothermal nanoagents for PA imaging and PTT applications. The photophysical properties and applications of organic/polymer photothermal nanoagents are summarized and compared in Table 1 . In the following, we firstly discuss the design strategies to enhance the PA imaging and PTT effect of both SPNs and small-molecule organic photothermal agents-encapsulated NPs, respectively. Then, we highlight the new emerging mechanism to enhance the PA imaging and PTT effects of organic/ polymer photothermal nanoagents based on "molecular motion in aggregates". At last, a brief summary is given along with a perspective in this field.

\section{DESIGN STRATEGIES FOR SPNs}

Semiconducting polymers (SPs) are optically active polymers with large $\pi$-conjugated aromatic or aromatic heterocyclic structures [49]. Owing to the intrinsic advantages such as large absorption coefficient, high photostability and excellent biocompatibility, SPs have emerged as one of the most popular photothermal agents for PA imaging and PTT [50]. Correspondingly, the regulation of the photophysical properties of SPs for enhanced in vivo PA imaging and PTT effects has gained large attention among researchers in the biomedical field.

\section{Molecular structure screening strategy}

Given the critical role of extinction coefficient in the photophysical property regulation, the molecular structure screening of the photothermal nanoagents with high extinction coefficients is an important approach to en- hance the PA imaging and PTT effect [33]. Recently, Rao and co-workers [51] developed two kinds of SPs, poly (cyclopentadithiophene-alt-benzoth-iadiazole) (SP1) and poly (acenaphthothieno-pyrazine-alt-benzodithiophene) (SP2) with different extinction coefficients by introducing different donor-acceptor $(\mathrm{D}-\mathrm{A})$ structures, which also endowed SP1 and SP2 with near-infrared (NIR) light absorbing characteristic (Fig. 1a). It is noteworthy that NIR light absorbing characteristic is also favorable to the enhanced PA imaging and PTT effect of photothermal agents in vivo, owing to its strong tissue penetration ability and high biosecurity [52,53]. To overcome the hydrophobicity of these two SPs, nanoprecipitation method was then introduced to afford water-dispersed SPNs, termed as SPN1 and SPN2, respectively. Due to the difference in the molecular structures, SPN1 showed 4.65times higher peak mass extinction coefficient than that of SPN2. Moreover, owing to the larger absorption coefficient, SPN1 also exhibited 5.2 and 7.1-times higher PA amplitude at the same mass concentration compared with high-performing single-walled carbon nanotubes and gold nanorods, respectively, which made SPN1 a better choice for in vivo PA imaging. With a small hydrodynamic diameter of $41 \mathrm{~nm}$, SPN1 demonstrated its ability to image the main lymph nodes in vivo of mice with a high PA signal to noise $(\mathrm{S} / \mathrm{N})$ ratio. Considering the critical role of reactive oxygen species (ROS) in the development of many life-threatening diseases which are often highly expressed in the disease lesions, the detection of ROS is very helpful in understanding the disease mechanisms and for their early diagnosis $[54,55]$. Therefore, an ROS-sensitive dye, IR775S, was then coupled with the oxidation-inert SPN1 to form the first NIR ratiometric PA imaging probe (RSPN) for ROS detection (Fig. 1b). The PA spectrum of RSPN exhibited three main peaks at 700,735 , and $820 \mathrm{~nm}$, with the first one corresponding to SP1 and the last two to IR775S. By calculating the PA700/ PA820 ratio of RSPN, the specific detection of exogenous $\mathrm{ONOO}^{-}$and $\mathrm{ClO}^{-}$was achieved (Fig. 1c). Cell experiments and in vivo mouse experiments also confirmed the dual-peak ratiometric detection capacity of RSPN for endogenously generated ROS. This study describes an important approach for enhanced PA signals of SPs with high extinction coefficients through molecular structure screening and a strategy for ROS detection in vivo via physical doping.

\section{Molecular structure engineering strategy}

Although the above-mentioned work confirmed that enhanced PA signals could be obtained by regulating the 


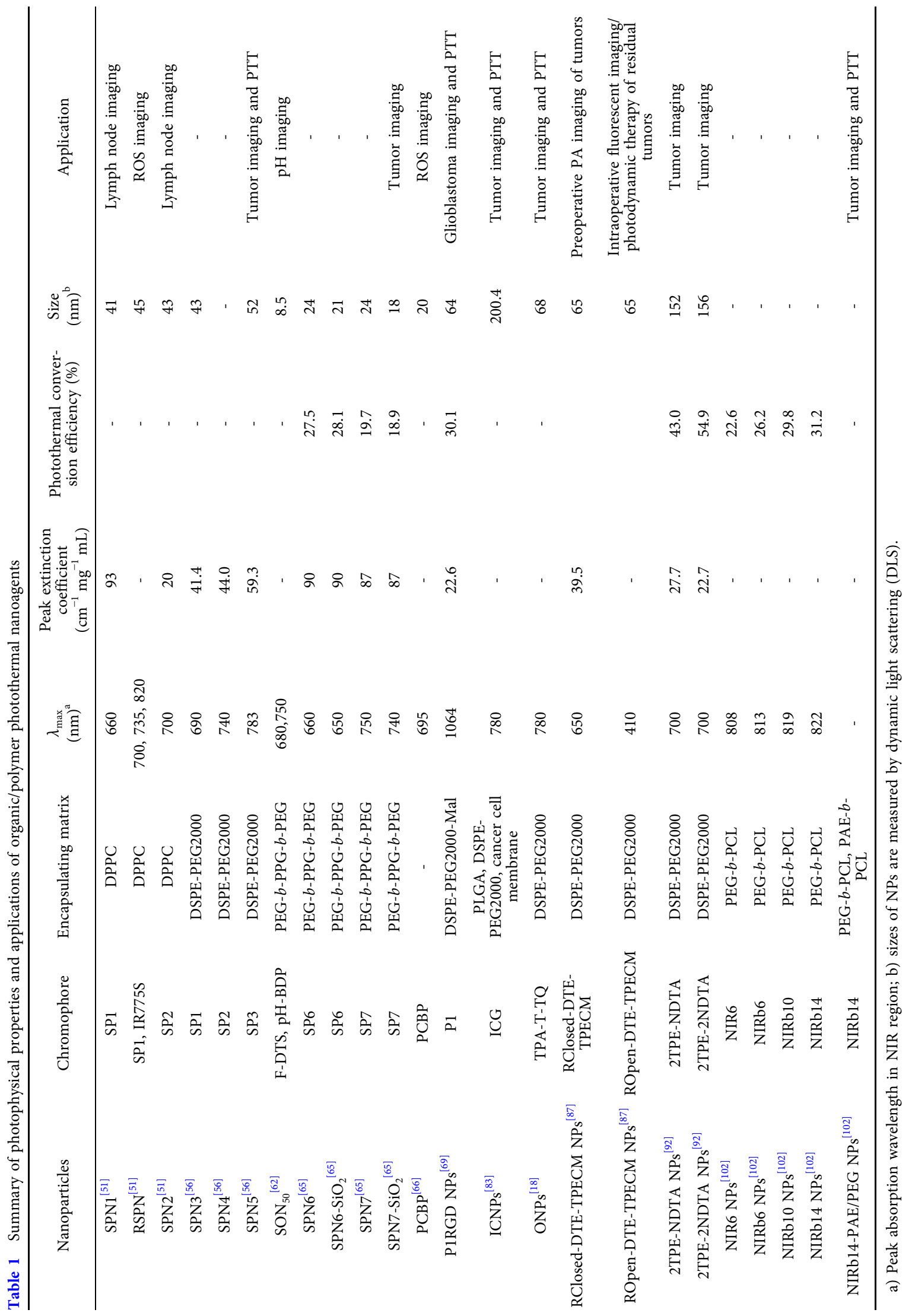




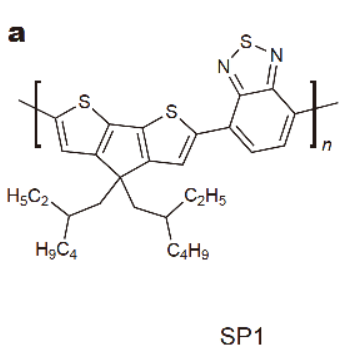

SP1

b

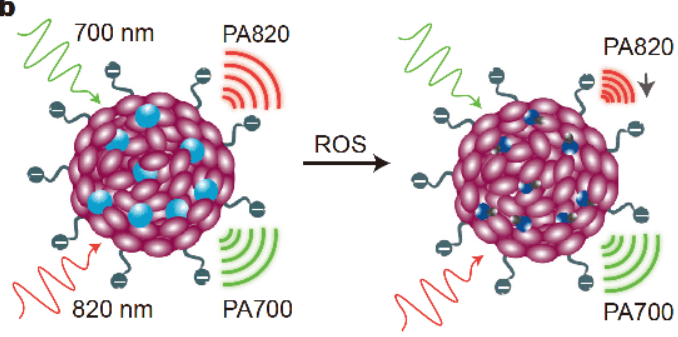

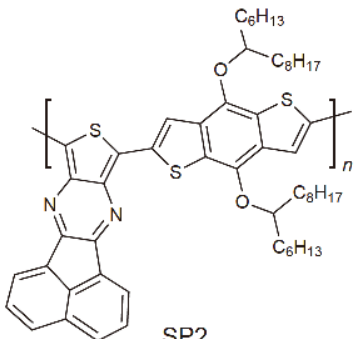

SP2

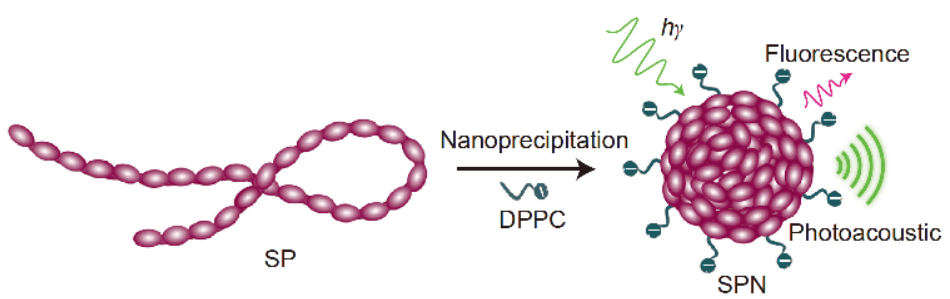

c 30

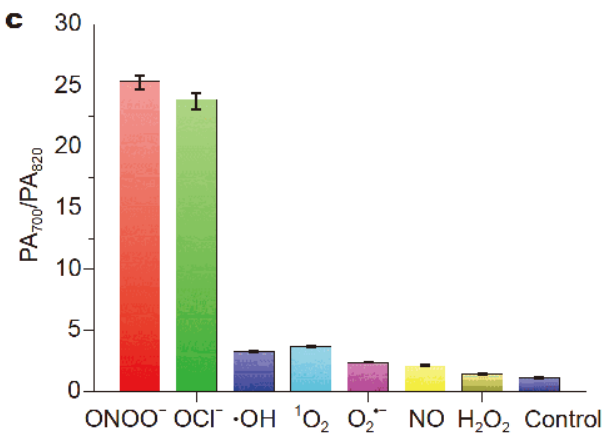

Figure 1 (a) Chemical structures of SP1 and SP2 and schematic diagrams of their preparation into NPs. (b) Proposed ROS sensing mechanism of RSPN (ratiometric SPN). (c) Ratios of PA amplitude of RSPN at $700 \mathrm{~nm}$ to that at $820 \mathrm{~nm}$ (PA700/PA820) in the presence of different ROS $\left(5 \mathrm{mmol} \mathrm{L}^{-1}\right)$. Reproduced with permission from Ref. [51]. Copyright 2014, Springer Nature.

molecular structures of photothermal agents, theoretical guidance was still lack in designing photothermal agents with enhanced PA imaging and PTT effect. To overcome this limitation, a molecular structure engineering approach was proposed by Liu and co-workers [56] to design high-performing SPs with $\mathrm{D}-\mathrm{A}$ structures. A planar donor, $4 H$-dithieno $\left[3,2-b: 2^{\prime}, 3^{\prime}-d\right]$ pyrrole (DTP) was copolymerized with three electron-deficient acceptors, benzothiadiazole (BT), pyridal $[2,1,3]$ thiadiazole $(\mathrm{PT})$, and diketopyrrolopyrrole (DPP) respectively to construct SP3-5 with an increasing $\mathrm{D}-\mathrm{A}$ strength in sequence (Fig. 2a). 1,2-Distearoyl-sn-glycero-3-phospho-ethanolamine- $N$-[amino(polyethylene glycol)-2000] (DSPE$\mathrm{PEG}_{2000}$ ), was then introduced as the amphipathic polymer matrix to afford SPN3-5 with good water dispersibility (Fig. 2b). Owing to the strong intramolecular charge transfer (ICT) introduced by high $\mathrm{D}-\mathrm{A}$ strength, SP3-5 have an increasing red-shifted absorption peak at 703,748 , and $858 \mathrm{~nm}$, respectively. In addition, benefiting from the rigid planar structures, these three SPs have long conjugation length and subsequent large extinction coefficients. The extinction coefficients of SPN4 and SPN5 at $780 \mathrm{~nm}$ are 1.6- and 2.2-times higher than that of SPN3. Moreover, the fluorescence quantum yields of SP3-5 are very low and decrease with the enhancing D-A strength. Since fluorescence emission, intersystem crossing (phosphorescence and/or ROS generation) and thermal deactivation are the main three dissipation pathways of the light excitation energy absorbed by photothermal agents [57-59], the quenched fluorescence emission thus contributes to amplifying PA signal ratios of SPN4 and SPN5 to SPN3 (2.8 and 5.8, respectively). On account of the best in vitro PA performance, SPN5 was selected for in vivo PA imaging of mouse tumor (Fig. 2c), with an extremely high $\mathrm{S} / \mathrm{N}$ ratio of 47 at $3.2 \mathrm{~mm}$ depth, while the $\mathrm{S} / \mathrm{N}$ ratio of indocyanine green (ICG, a commonly used organic small molecule photothermal agent) was 5 . Owing to the excellent photothermal conversion capability, SPN5 also exhibited an extraordinarily low half maximal inhibitory concentration $\left(\mathrm{IC}_{50}\right)$ value of $0.88 \mu \mathrm{g} \mathrm{mL}$ upon 5-min laser irradiation at $808 \mathrm{~nm}\left(0.8 \mathrm{~W} \mathrm{~cm}^{-2}\right)$ and an impressive in vivo PTT effect with U87 tumor totally eliminated. This study highlights a general molecular structure engineering design guideline to enhance the PA imaging and PTT effect of organic photothermal agents by increasing their $\mathrm{D}-\mathrm{A}$ strength, which simultaneously aggrandizes their extinction coefficients and non-radiative decay efficiencies.

\section{Nanoengineering approach}

Thanks to the guidance of molecular structure engineering approach, lots of organic/polymer photothermal nanoagents with outstanding photothermal conversion capability have been developed, realizing better applications in PA imaging and PTT $[60,61]$. However, molecular structure engineering approach also 

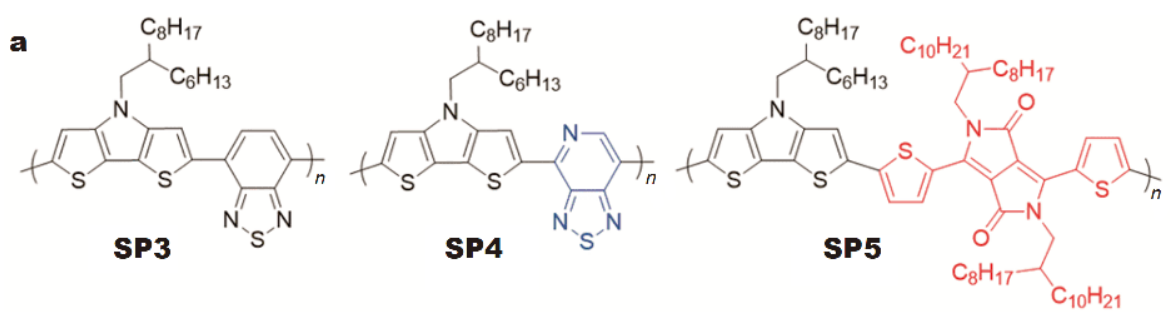

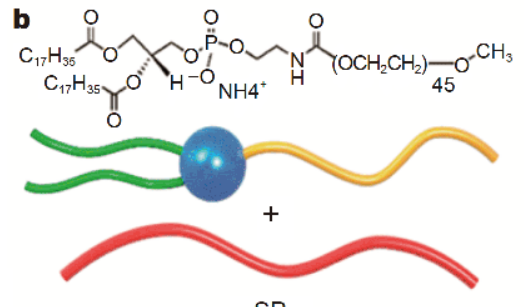

SP

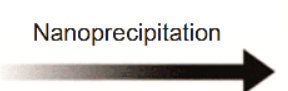

C

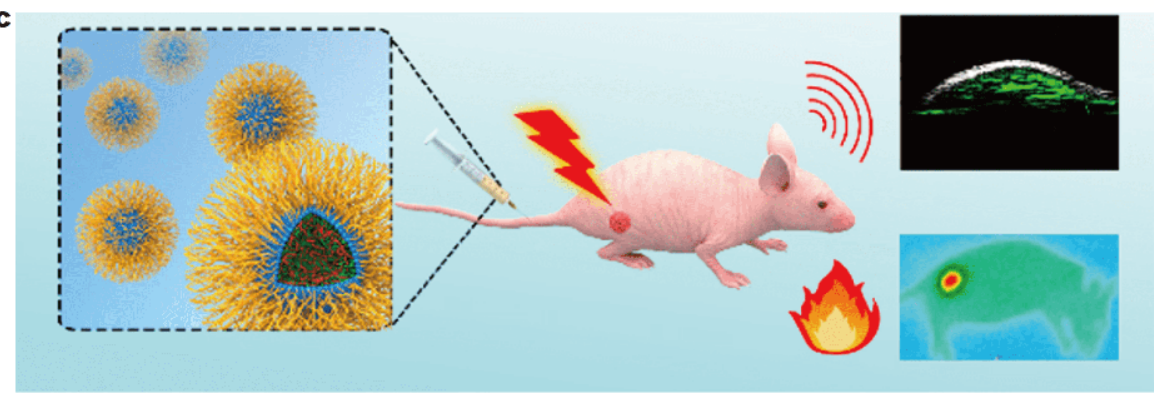

Figure 2 (a) Molecular structures of SP3-5. (b) Schematic of the preparation of SPNs through nanoprecipitation. (c) Schematic diagram of the application of SPNs in the PA imaging and PTT of tumors in live mice. Reproduced with permission from Ref. [56]. Copyright 2017, American Chemical Society.

encounters some limitations, such as difficult synthesis and time-consuming screening process. Thus, simple and effective means to improve the PA imaging and PTT effect of photothermal agents are still highly demanded. Recently, Pu and co-workers [62] reported a convenient intraparticle nanoengineering approach to amplify the PA signals of photothermal agents for in vivo $\mathrm{pH}$ detection, considering the close association of aberrant $\mathrm{pH}$ with many diseases ranging from inflammation to cancer $[63,64]$. By nanodoping of a semiconducting oligomer (SO) and a boron-dipyrromethene dye (pH-BDP), which is $\mathrm{pH}$-sensitive due to the protonation of the hydroxyl group upon acidification, an activatable semiconducting oligomer nanoprobe (SON) was obtained utilizing amphiphilic poly(ethyleneglycol)-block-poly(propyleneglycol)-block-poly(ethyleneglycol) (PEG- $b$-PPG- $b$-PEG) as the encapsulating matrix (Fig. 3a). In addition to be a $\mathrm{pH}$ indicator, $\mathrm{pH}-\mathrm{BDP}$ also acts as a PA enhancer. Due to the higher electron affinity and ionization potential of SO with a low molecular weight (F-DTS) compared with that of pH-BDP, photoinduced electron transfer (PET) was induced between those two optical components, resulting in quenched fluorescence of F-DTS and consequent amplifying PA brightness of SONs (Fig. 3b). Along with a decreasing $\mathrm{pH}$, the absorption spectra of SONs exhibited a distinct decreasing peak at $750 \mathrm{~nm}$, while the peak at $680 \mathrm{~nm}$ changed slightly (Fig. 3c). Owing to the direct association of PA brightness with the absorption coefficients of photothermal agents, this spectral response toward $\mathrm{pH}$ endows SONs with dual-peak ratiometric detection ability for $\mathrm{pH}$ (PA680/PA750). To achieve the optimal PA imaging effect, the relationship between the $\mathrm{PA}$ brightness of SONs and the doping ratio of $\mathrm{pH}-\mathrm{BDP}$ was also researched. With the increasing doping ratio of $\mathrm{pH}-\mathrm{BDP}$, the fluorescence of SONs gradually decayed, leading to a continuous increasing PA signal until the doping ratio of $\mathrm{pH}-\mathrm{BDP}$ to be $75 \%\left(\mathrm{SON}_{75}\right) . \mathrm{SON}_{50}$ was then selected for the subsequent PA imaging owing to the highest $\mathrm{S} / \mathrm{N}$ ratio and the close PA brightness to $\mathrm{SON}_{75}$. The ratiometric PA signal of $\mathrm{SON}_{50}$ solution at $\mathrm{pH} 5.5$ is 


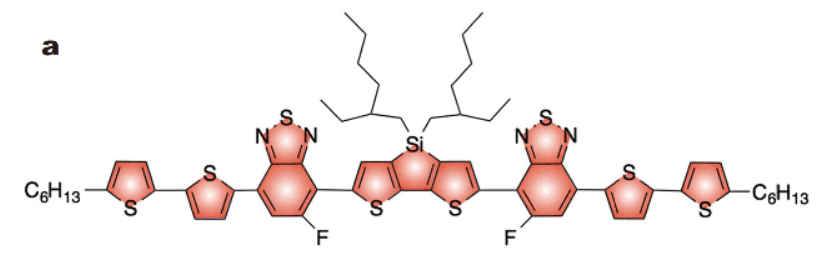

SO: F-DTS

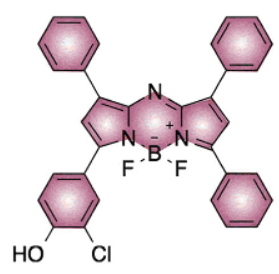

$\mathrm{pH}$ indicator: $\mathrm{pH}-\mathrm{BDP}$

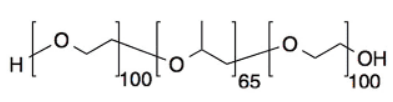

PEG- $b$-PPG- $b$-PEG
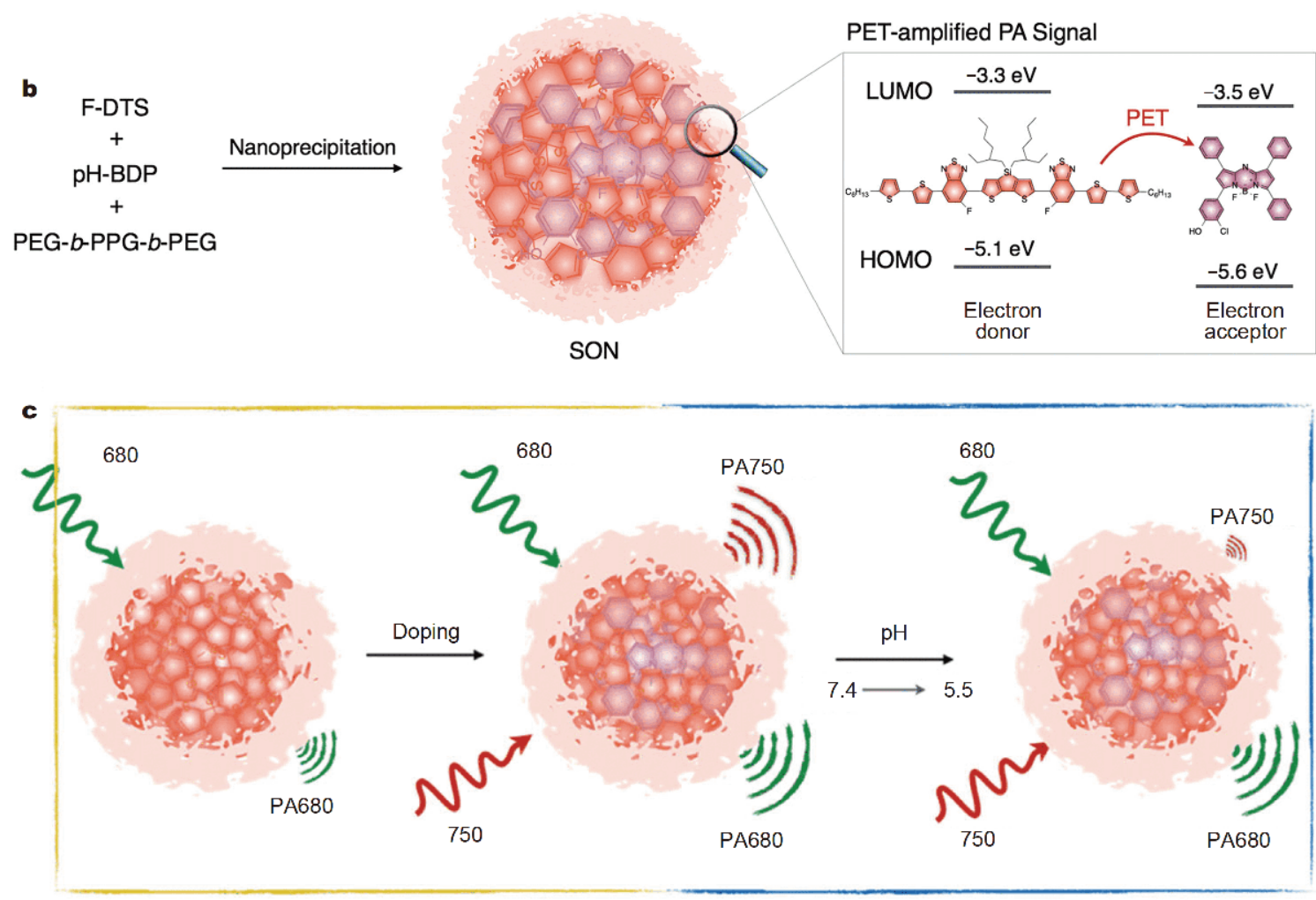

PA Amplification

Ratiometric sensing

Figure 3 (a) Molecular structures of F-DTS, pH-BDP and PEG-b-PPG- $b$-PEG. (b) Schematic of the preparation of SONs and the PET mechanism between F-DTS and pH-BDP. (c) Schematic illustration of the PA amplification induced by doping and the mechanism for $\mathrm{pH}$ detection. Reproduced with permission from Ref. [62]. Copyright 2016, WILEY-VCH Verlag GmbH \& Co. KGaA, Weinheim.

1.8- and 3.1-times higher than that at $\mathrm{pH} 6.4$ and 7.4, respectively. Especially, there existed a good linear correlation between $\mathrm{pH}$ and the ratiometric $\mathrm{PA}$ signal of $\mathrm{SON}_{50}$ with $R^{2}=0.991$ in the range of $\mathrm{pH} 7.4$ to 5.5 , endowing $\mathrm{SON}_{50}$ with an excellent capacity to quantify the $\mathrm{pH}$ of tumors in vivo. This new intraparticle nanoengineering strategy through modulating the energy levels of binary components, which hence increases the non-radiative decay efficiencies, provides a facile approach to amplify the PA imaging and PTT effect of organic/polymer photothermal agents.

However, in spite of the convenience and effectiveness, intraparticle nanoengineering strategy also suffers from its own limitation: this strategy is only applied to fluorescent photothermal nanoagents, and the fluorescence of photothermal nanoagents quenches after doping, which suppresses the potential for simultaneous fluorescence imaging. To address this restriction, $\mathrm{Pu}$ and co-workers [65] further developed a general surface nanoengineering strategy to concurrently enhance the fluorescence and PA signals of photothermal nanoagents. By coating of tetraethyl orthosilicate (TEOS) on the surface of SPN6, which was prepared by the assembly of fluorescent poly(cyclopentadithiophene-alt-benzothiadiazole) (SP6) with PEG- 
$b$-PPG- $b$-PEG, multilayered SPN6-SiO ${ }_{2}$ with a thin optically inner silica shell on the surface was constructed. Owning to the critical role of the molecular structures of SPs in the regulation of the absorption and photothermal conversion efficiencies of SPNs, SPN6 and $\mathrm{SPN}_{-}-\mathrm{SiO}_{2}$ have similar absorption spectra curves and maximum photothermal temperatures. However, the temperature of SPN6 increased lower than that of $\mathrm{SPN}^{-\mathrm{SiO}_{2}}$ in the initial stage of continuous laser irradiation, indicating the higher heat interfacial conductance and hence the smaller interfacial heat diffusion resistance of silica to water as compared with that of the SP6 core to water. Since the heat diffusing rate of NPs to water also plays an important role in the control of the PA performance of photothermal nanoagents, $\mathrm{SPN} 6-\mathrm{SiO}_{2}$ with higher heat diffusing rate exhibits a 1.4 higher PA amplitude than uncoated SPN6 at the same SP6 mass concentration. Specially, due to the effective suppression of the interactions between SP6 molecules and water caused by silicon coating, $\mathrm{SPN} 6-\mathrm{SiO}_{2}$ also shows a 5.22-times elevated fluorescence quantum yield than SPN6. This characteristic of surface nanoengineering strategy is different from that of the above intraparticle nanoengineering strategy, wherein the PA brightness is amplified along with a quenched fluorescence. To demonstrate the universality of this surface nanoengineering strategy, a non-fluorescence SP analogue, poly(cyclopentadithiophene-alt-diketopyrrolopyrrole) (SP7), was also utilized to prepare the SPNs. Similar to SP6, silica-coated SPN7-SiO ${ }_{2}$ also performed a higher PA amplitude than the corresponding uncoated SPN7, affirming the universality of this surface nanoengineering strategy for enhanced PA signals of photothermal nanoagents. By conjugation of cyclo(Arg-GlyAsp-D-Phe-Lys(mpa)) (c-RGD) peptides to the surface of SPN1-SiO ${ }_{2}$, the proof-of-concept application of the surface nanoengineered SPN was demonstrated for targeted PA imaging of tumor in vivo. This study highlights a general surface nanoengineering strategy to concurrently amplify the PA brightness and fluorescence of organic/ polymer photothermal nanoagents by increasing the heat interfacial conductance from the nanoagents to water and suppressing the interactions between the fluorescent photothermal molecules and water.

\section{Stimuli-controlled NP regrowth}

Stimuli-controlled NP regrowth is another efficient approach to activate and enhance the PA imaging and PTT effect of photothermal agents. For instance, $\mathrm{Pu}$ and co-workers [66] reported a semiconducting PA probe with target-enhanced signal for in vivo ROS detection. By covalent linking phthalocyanine and PEG with phenylboronic acid pinacol ester group, a ROS-responsive amphiphilic SP (PCBP) was synthesized. Owing to the excellent amphiphilicity, PCBP formed stable NPs with small average hydrodynamic sizes of $20 \mathrm{~nm}$. While under ROS-stimulated conditions including $\mathrm{H}_{2} \mathrm{O}_{2}$ and $\mathrm{ONOO}^{-}$, the phenylboronic acid pinacol ester group of PCBP selfimmolated to cleave hydrophilic PEG from PCBP, leaving hydrophobic phthalocyanine behind, which subsequently self-assembled and regrew into larger NPs with large average hydrodynamic sizes of $350 \mathrm{~nm}$ (Fig. 4). Owing to the accelerated heat transfer within large NPs compared with corresponding small NPs [58,67-69], such an ROSresponsive regrowth of PCBP eventually generated amplified PA signals for ROS detection. In vitro PA experiment showed a linear relationship between PA amplitude of PCBP solution and the concentration of $\mathrm{H}_{2} \mathrm{O}_{2}$ or $\mathrm{ONOO}^{-}$, confirming the feasibility of PCBP for the quantification of $\mathrm{H}_{2} \mathrm{O}_{2}$ and $\mathrm{ONOO}^{-}$. In contrast, the PA amplitude of PCBP changed slightly upon other ROS including ${ }^{\circ} \mathrm{OH},{ }^{1} \mathrm{O}_{2}, \mathrm{ClO}^{-}$and $\mathrm{O}_{2}{ }^{--}$. Moreover, the PA amplitude of the control probe without ROS linker changed slightly for all ROS. Benefitted from the prolonged retention time and enhanced PA signals of regenerate large NPs, PCBP had an outstanding performance in the detection of the ROS level at the tumor sites in vivo. This study provides a supramolecular approach to enhance the PA signals of organic/polymer photothermal nanoagents by stimuli-controlled NP regrowth, which increases the rate of heat transfer within nanoagents.

\section{Extending absorption wavelength to NIR-II window}

Moreover, since photons need to penetrate tissues to supply energy to photothermal agents during in vivo application, extending the absorption wavelength with stronger tissue penetration ability is also an effective way to enhance the PA imaging and PTT effect of photothermal agents. Recently, Liu and co-workers [69] developed a kind of SPNs with strong absorption in the second near-infrared (NIR-II) window for enhanced PA imaging and PTT of brain tumor. By alternatively polymerization of an electron-rich donor and an electrondeficient acceptor, a planar donor-acceptor structured SP (P1) with a broad NIR-II absorption peak near $1064 \mathrm{~nm}$ was constructed (Fig. 5). To overcome the hydrophobicity of P1, amphipathic DSPE-PEG ${ }_{2000}$-Mal was then introduced to afford P1 NPs with good water dispersibility. In vitro tissue penetration experiments demonstrated the superior tissue penetration ability of $1064 \mathrm{~nm}$ laser over 


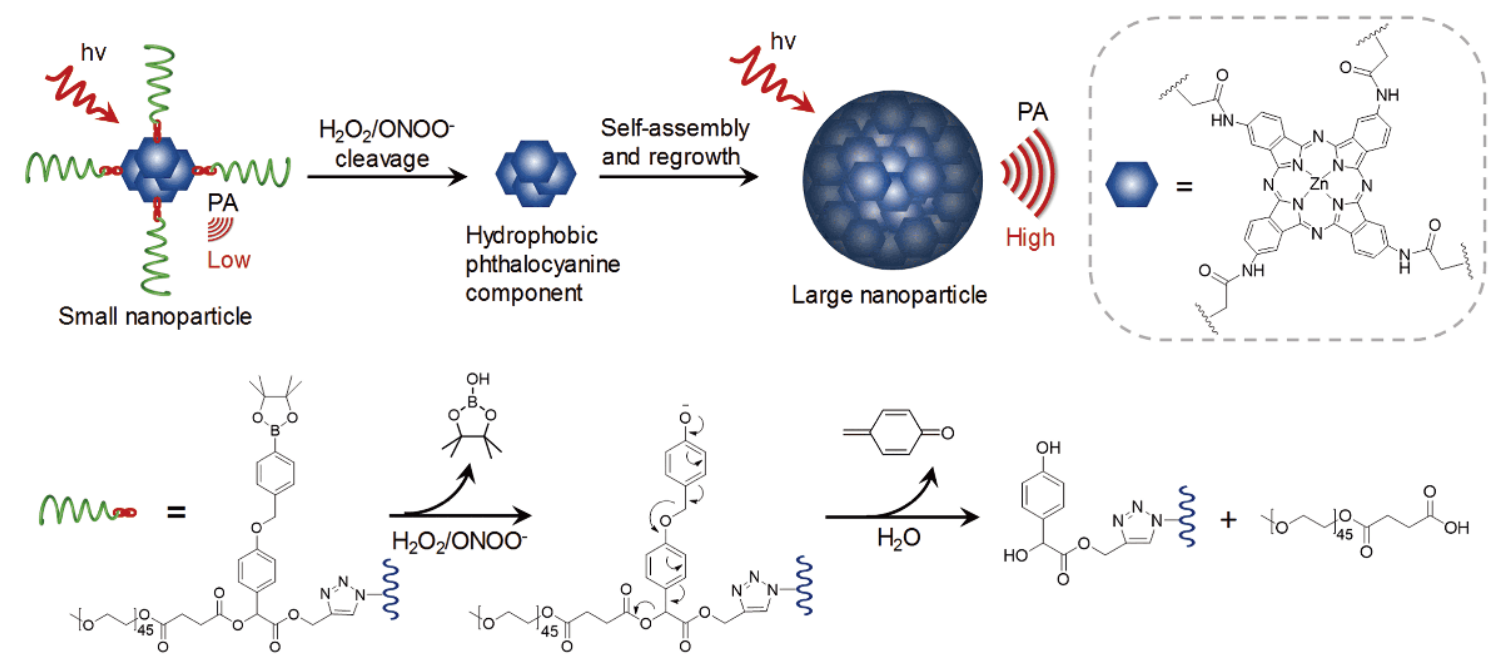

Figure 4 Schematic illustration and mechanism of the ROS-induced regrowth of PCBP NPs for enhanced PA imaging. Reproduced with permission from Ref. [66]. Copyright 2017, WILEY-VCH Verlag GmbH \& Co. KGaA, Weinheim.

$808 \mathrm{~nm}$ laser, which was the commonly used NIR-I laser, with $46 \%$ light energy lost for $1064 \mathrm{~nm}$ laser and $70 \%$ lost for $808 \mathrm{~nm}$ through $2 \mathrm{~mm}$ chicken tissue. Owing to the strong tissue penetration capacity of NIR-II laser and high absorption of P1 NPs in the NIR-II window, P1 NPs exhibited excellent deep tissue imaging performance with PA signals clearly detected under even $8 \mathrm{~mm}$ chicken tissues upon $1064 \mathrm{~nm}$ pulsed laser irradiation [70]. In order to apply P1 NPs to the diagnosis and treatment of brain glioblastoma, which disheartened many phototheranostic agents with short wavelength absorption due to the severely attenuate light caused by dense skull and scalp, the surfaces of P1 NPs were further decorated with c-RGD peptides, affording P1RGD NPs with dual-tar- geting ability for endothelial cells in the glioblastoma angiogenic vasculatures and glioblastoma cells as well. Benefitted from the enhanced tumor cellular uptake and the strong absorption in NIR-II window, P1RGD NPs showed an outstanding performance in the PA imaging of orthotopic glioblastoma with $\mathrm{S} / \mathrm{N}$ ratio to be 90 , while the $\mathrm{S} / \mathrm{N}$ ratio of P1 NPs was 61 , both far higher than the S/N threshold of 5 to clearly distinguish glioblastoma from surrounding tissues [38,71]. Moreover, P1RGD NPs also performed well in PA image-guided PTT, with effective tumor growth suppression upon low safety therapeutic power of $1064 \mathrm{~nm}$ laser, while the power of $808 \mathrm{~nm}$ laser utilized in the reported glioblastoma PTT was almost far beyond the safety limit $[72,73]$. This study highlights an

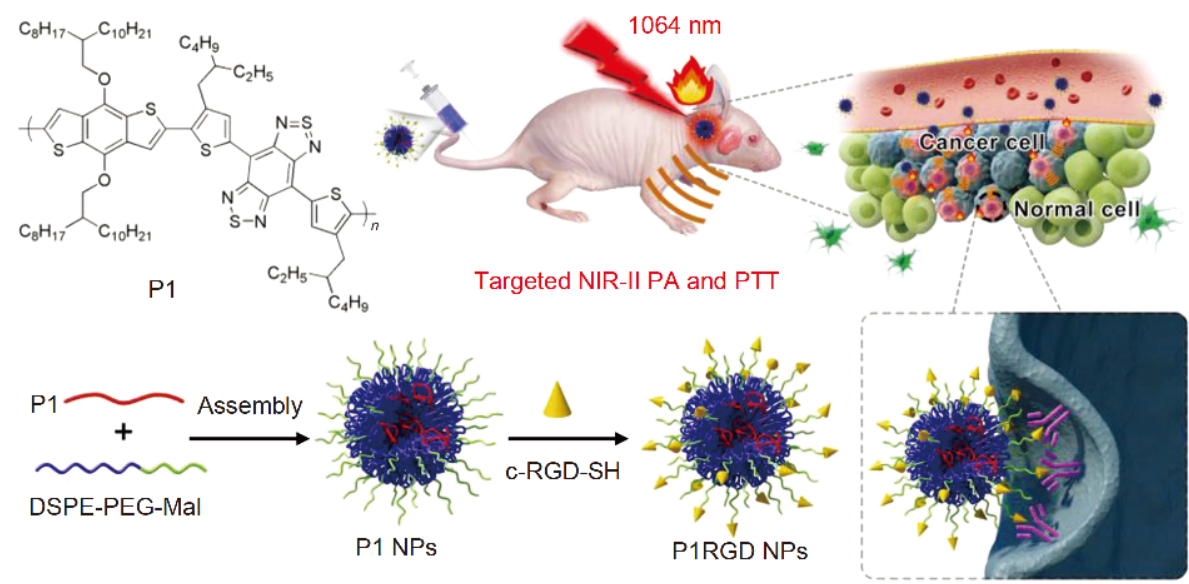

Figure 5 Schematic illustration of the preparation of P1RGD NPs and the in vivo PA imaging and PTT of brain tumors. Reproduced with permission from Ref. [69]. Copyright 2018, WILEY-VCH Verlag GmbH \& Co. KGaA, Weinheim. 
effective approach to enhance the in vivo PA imaging and PTT effect of organic/polymer photothermal nanoagents by extending the absorption wavelength to the NIR-II window, which increases the light energy harvested by photothermal nanoagents at deep tissues. This approach is believed to bring about a revolution in organic/polymer photothermal nanoagents and promote their better application in PA imaging and PTT.

\section{DESIGN STRATEGIES FOR SMALL- MOLECULE ORGANIC PHOTOTHERMAL AGENTS-ENCAPSULATED NPs}

Small-molecule organic photothermal agents have long been a research hotspot in the biomedical field due to their rich variety. However, small-molecule organic photothermal agents often suffer from poor hydrophilicity, short blood circulation time and low tumor targeting ability, which severely restrict their in vivo PA imaging and PTT effect [74]. To overcome this limitation, amphiphilic co-polymers have been introduced to encapsulate small-molecule organic photothermal agents into the hydrophobic core of self-assembly NPs, endowing small-molecule organic photothermal agents with high hydrophilicity and prolonged half-life in serum, as well as active and passive tumor-targeting capability via ligand-receptor interactions and EPR effect, respectively [75-77]. These amphiphilic polymers are generally composed of a hydrophobic portion and a hydrophilic portion (e.g., PEG, and zwitterionic material, etc.), with the former forming a stable hydrophobic core, while the latter forming a hydration layer to reduce nonspecific serum protein adsorption [78,79]. Benefitted from the prolonged blood circulation time resulting from the reduced serum protein adsorption as well as the EPR effect, organic/polymer photothermal NPs exhibited an enhanced tumor accumulation compared with small-molecule organic photothermal agents themselves. However, on account of the exogeneity, NPs were still largely recognized by the immune system and mostly cleared by the liver and kidney [80-82], resulting in a relatively low NPs accumulation in the tumor sites and hence a nonideal PA imaging and PTT effect. Therefore, designing biomimetic organic/polymer photothermal NPs with reduced accumulation in the liver and kidney and increased enrichment at the tumor sites could be an effective approach to enhance the PA imaging and PTT effect of tumor in vivo.

Increasing tumor accumulation via biomimetic approach Recently, Cai and co-workers [83] reported a kind of cancer cell membrane-coated photothermal NPs (ICNPs) based on the fusion of PEGylated cell membrane vesicles self-assembled by the extracted MCF-7 cancer cell membranes and DSPE-PEG on the surface of ICG-loaded poly(lactic-co-glycolic acid) (PLGA) cores (Fig. 6a). Owing to the outstanding disguise capacity of cancer cell membranes and resistance property to protein adsorption of PEG, ICNPs exhibited an extremely long blood circulation time, with the $\mathrm{AUC}_{24 \mathrm{~h}}$ (the area under the blood drug concentration-time curve over $24 \mathrm{~h}$ ) of ICNPs 11.7times higher than free ICG. Importantly, in vivo biodistribution experiment showed a much lower accumulation of ICNPs in the liver and kidneys than the corresponding ICG NPs (INPs) without cell membrane coating. In synergy with the homologous active targeting ability and passive targeting capability via the EPR effect, this reduced interception of the liver and kidney gave rise to 3.1- and 4.75-times higher tumor accumulation amount of ICNPs compared with INPs and free ICG, respectively. Benefitted from the highest tumor accumulation, ICNPs performed the best in the dual-modal imaging (fluorescence and PA imaging) and PTT of in vivo tumors among the three photothermal agents (Fig. 6b). This study describes a general approach to enhance the PA imaging and PTT effect of tumors in vivo by designing biomimetic organic/polymer photothermal nanoagents with increased accumulation at the tumor sites.

\section{Increasing the stability of small-molecule organic photothermal agents}

Compared with nanoengineering approach, developing organic small molecule photothermal agents with high photothermal conversion efficiencies and high stability is more effective to improve the overall in vivo PA imaging and PTT effect of photothermal NPs. In spite of possessing high photothermal conversion efficiencies, the stability of most small-molecule organic photothermal agents is poor, leading to subdued PA imaging and PTT effect. Recently, Tang, Ding and co-workers [18] developed a highly stable small-molecule organic photothermal agent (TPA-T-TQ) with NIR light-absorbing D-A structure (Fig. 7a). Amphiphilic DSPE-PEG ${ }_{2000}$ was further introduced to afford water-soluble TPA-T-TQ organic NPs (ONPs) with an average diameter of about $68 \mathrm{~nm}$. Compared with ICG itself and ICG NPs, TPA-T-TQ ONPs exhibited the highest photothermal stability, with a nearly constant absorption spectrum of TPA-T-TQ ONPs and a sharply decreasing maximal absorption intensity of ICG and ICG NPs after $15 \mathrm{~min} 808 \mathrm{~nm}$ laser irradiation (Fig. 7b). Anti-photobleaching experiment showed a 
a
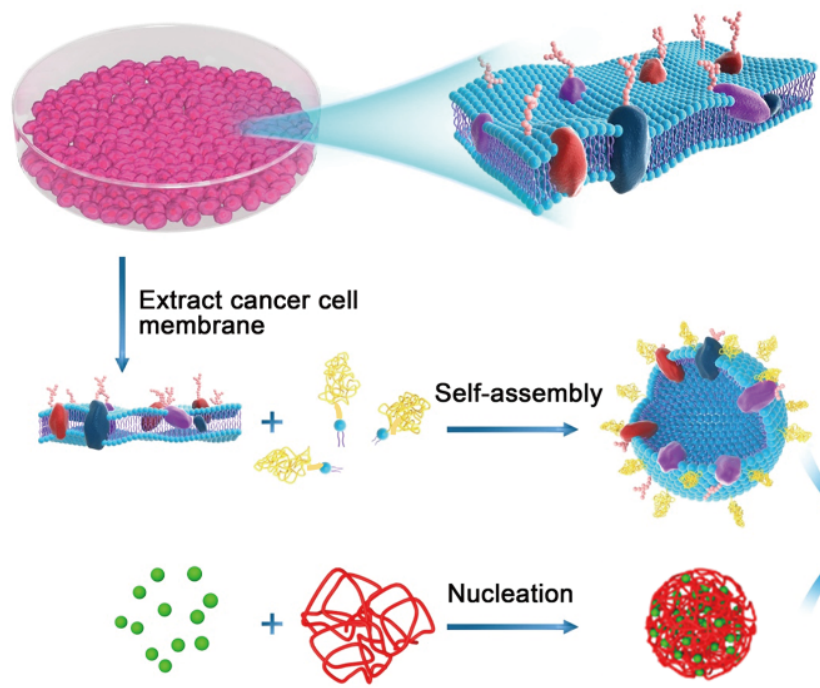

Nucleation
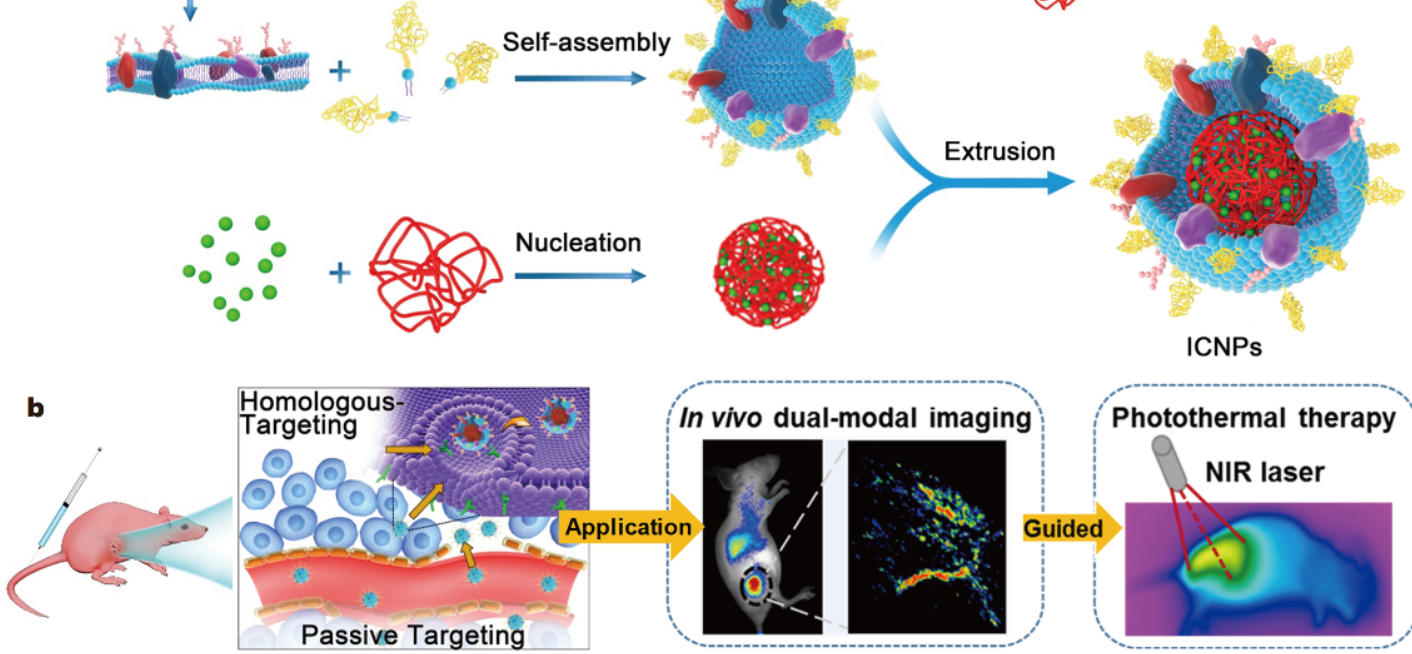

ICNPs

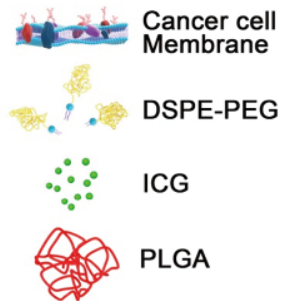

b

Figure 6 Schematic of the preparation procedure of homologous-targeting ICNPs (a) and their application in the dual-modal imaging guided PTT (b). Reproduced with permission from Ref. [83]. Copyright 2016, American Chemical Society.

negligibly changed photothermal conversion ability of TPA-T-TQ ONPs after five cycles of heating and cooling processes, and a $20 \%$ temperature elevation of the original value after two heating-cooling circles of ICG and ICG NPs, confirming the best photobleaching resistance of TPA-T-TQ ONPs among these three photothermal agents (Fig. 7c). Due to the overexpression of reactive oxygen/nitrogen species (RONS) in many disease lesions including inflammation and cancer, the physiological stability of small-molecule organic photothermal agents against RONS is also important in the diagnosis and treatment of RONS-associated diseases. Among these three photothermal agents, TPA-T-TQ ONPs exhibited the highest stability upon the addition of $\mathrm{ONOO}^{-}$and ${ }^{\circ} \mathrm{OH}$, two main kinds of RONS (Fig. 7d). Owing to the high photothermal stability and strong photobleaching/ RONS resistance, as well as large photothermal conversion efficiency, TPA-T-TQ ONPs exhibited an impressive performance in the PA imaging and PTT effect in vivo. This study provides a general approach to enhance the in vivo PA imaging and PTT effect of organic/polymer photothermal nanoagents by increasing the stability of small-molecule organic photothermal agents, which es- sentially maintains nearly constant extinction coefficients and photothermal conversion efficiencies in in vivo applications.

\section{Function-transformable strategy}

In spite of the advantage of deep imaging depth, PA imaging also has insufficient sensitivity when used alone. In recent years, due to the complementarity of PA imaging and fluorescence imaging, materials for dual-modality PA and fluorescence imaging, which integrates the merits of high spatial resolution of PA imaging with high sensitivity of fluorescence imaging, have attracted great attention among researchers [84-86]. However, according to Jablonski diagram, fluorescence emission, intersystem crossing (phosphorescence and/or ROS generation) and thermal deactivation are the main three dissipation pathways of the light excitation energy absorbed by chromophores. This competitive relationship between fluorescence emission and thermal deactivation determines that fluorescence and PA imaging of aforementioned materials could not be maximized at the same time. Therefore, it is urgently desired to develop intelligent materials featuring adaptive $\mathrm{PA} /$ fluorescence 


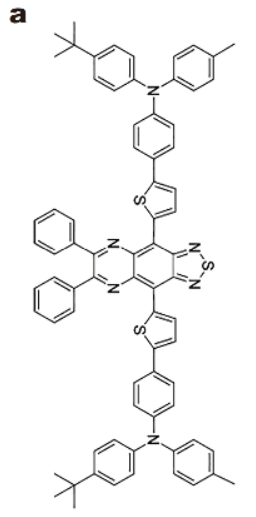

TPA-T-TQ
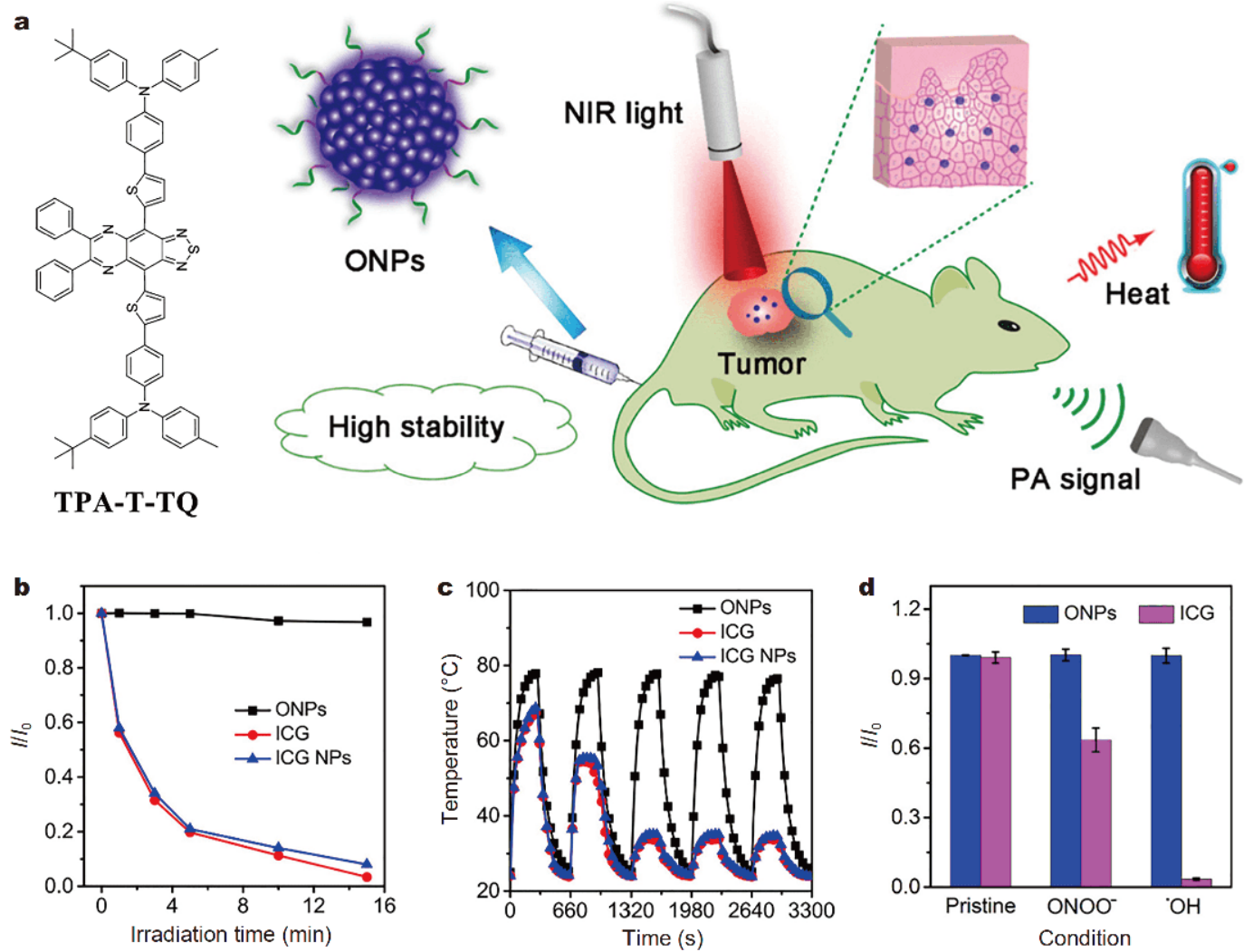

Figure 7 (a) Schematic of the application of ONPs in PA imaging and PA imaging-guided PTT. (b) The ratios of the maximal absorption intensity of ONPs/ICG/ICG NPs after and before laser irradiation $\left(I / I_{0}\right)$ various irradiation time. (c) Anti-photobleaching property of ONPs/ICG/ICG NPs during five heating-cooling cycles. (D) The ratios of the maximal absorption intensity of ONPs and ICG in the presence and absence of different kinds of RONS. Reproduced with permission from Ref. [18]. Copyright 2017, American Chemical Society.

imaging functions with utmost efficacies.

To meet this demand, Tang, Ding and co-workers [87] developed a function-transformable optical agent (DTETPECM) consisting of a photo-controllable dithienylethene (DTE) core and two ambient 2-(1-(4-(1,2,2-triphenylvinyl)phenyl)ethylidene) malononitrile (TPECM) units. DTE-TPECM has two isomers with the ring in the core open (ROpen-DTE-TPECM) or closed (RClosedDTE-TPECM), which reversibly switches upon external UV/visible light irradiation (Fig. 8a). In the ring-closing form, owing to the synergy between the intramolecular energy transfer from TPECM to DTE and the intermolecular interactions arising from the relatively planar geometric structure, the NIR-absorbed energy of RClosedDTE-TPECM is mainly dissipated by thermal deactivation with no detectable fluorescence emission and pimping ROS generation (Fig. 8b). This tilt of the photophysical energy balance to thermal deactivation is greatly favorable for PA imaging. While upon visible light irradiation, the ring in the core opens to yield ROpen-DTE-TPECM with disappeared intramolecular energy transfer and reduced intermolecular interactions result from the more twisted $3 \mathrm{D}$ geometric structure. These tremendously suppress the thermal deactivation, leading to utmost absorbed energy flowing to fluorescence emission and ROS generation (Fig. 8c). In order to make DTE-TPECM applied in image-guided cancer surgery, amphiphilic maleimide-bearing lipid-PEG2000 and YSAYPDSVPMMS peptide were then introduced to afford tumor-targeting DTE-TPECM NPs with good water solubility. Thanks to the excellent tumor-targeting and function-transformable property, DTE-TPECM NPs exhibited an excellent performance in the preoperative PA imaging via RClosed-DTE-TPECM NPs and intraoperative fluorescent imaging/photodynamic therapy of residual tumors via ROpen-DTETPECM NPs, significantly reducing the risk of tumor recurrence. This study highlights a function-transformable strategy based on molecular structural transformations to maximize the PA and fluorescence imaging efficiencies of optical agents on demand. 

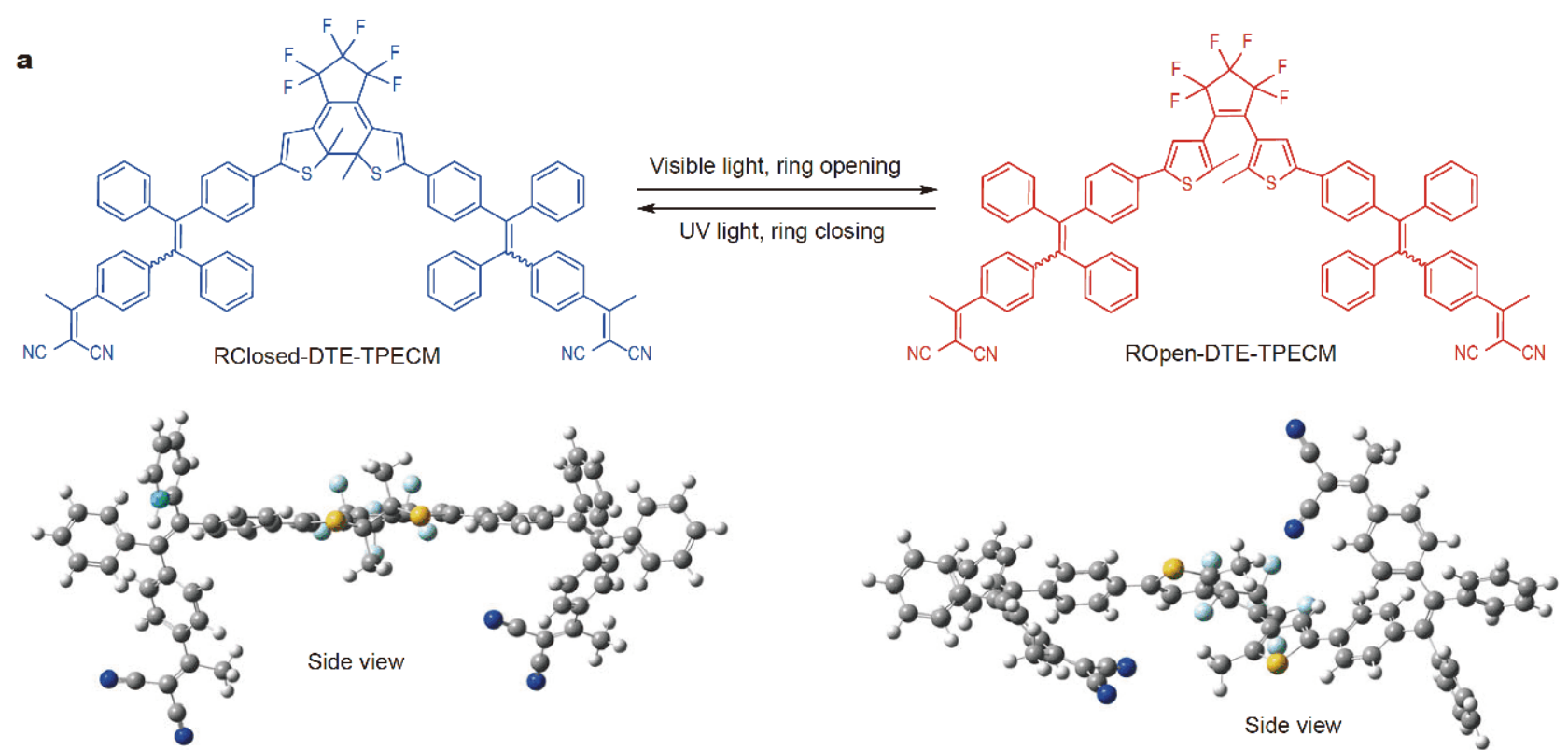

b

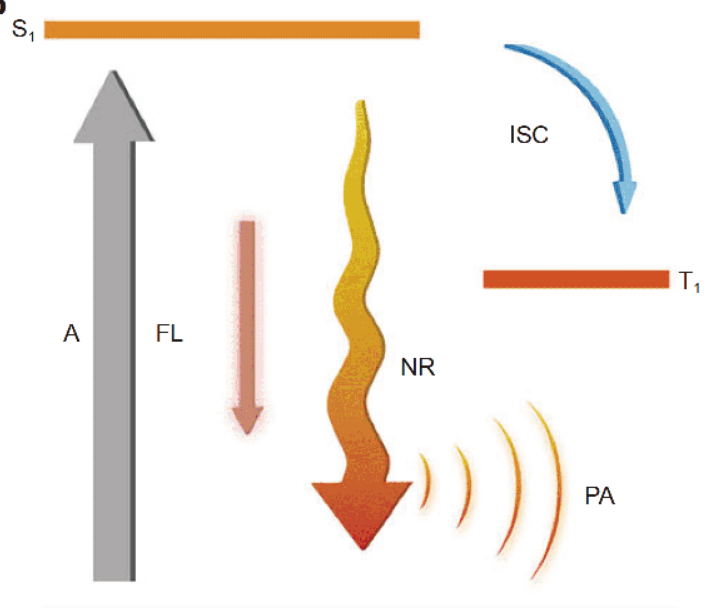

c

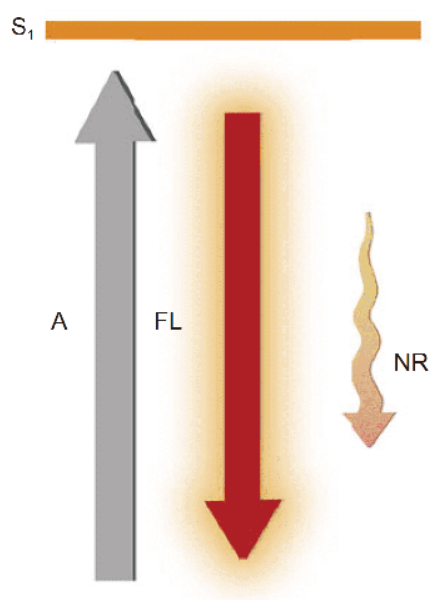

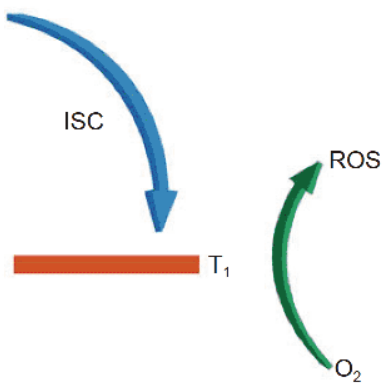

Figure 8 (a) Reversibility photo-controlled molecular structure of DTE-TPECM and its optimized geometric structure in the ring-closing/open form. $(b, c)$ Jablonski diagrams illustrating the photophysical processes of RClosed-DTE-TPECM (b) and ROpen-DTE-TPECM (c) upon laser irradiation. Reproduced with permission from Ref. [87]. Copyright 2018, Springer Nature.

\section{NEW EMERGING MECHANISM FOR ADVANCED ORGANIC/POLYMER PHOTOTHERMAL NANOAGENTS}

Summarizing the above-mentioned design strategies that have achieved enhanced PA imaging and PTT effects, they are all essentially based on the strong intermolecular interactions among photothermal molecules with planar structure. However, these strong intermolecular interactions impede other channels of thermal deactivation at the same time. Thus, new molecular design concepts to advanced photothermal agents with efficient thermal deactivation for enhanced PA imaging and PTT effects are still highly desirable. Recently, excited-state intramolecular motion has received increasing attention due to its capability of converting optical energy to mechanical energy or luminescence [88-90]. In addition, because of the theoretical feasibility of heat generation, excited-state intramolecular motion also has great potential for the development of advanced photothermal agents. However, this possibility has been rarely explored so far. Moreover, although active intramolecular motion of molecular rotors and vibrators has been achieved in 
solution, it is greatly attenuated in the aggregated or solid state, which significantly restricts its application in nanomaterials [91].

\section{Intramolecular motion-induced photothermy}

To address this issue, Tang, Ding and co-workers [92] developed two NIR-absorbing organic small molecule photothermal agents, 2TPE-NDTA and 2TPE-2NDTA, by Suzuki-Miyaura coupling reaction between the boric acid of tetraphenylethylene (TPE) and dibrominated naphthalene diimide-fused 2-(1,3-dithiol-2-ylidene) acetonitrile (NDTA)/2NDTA with long alkyl chains, respectively. In the aggregated state, owing to the intermolecular spatial isolation arising from the long alkyl chains, the rings of TPE are expected to rotate freely in the created rooms. To verify this concept, solid-state nuclear magnetic resonance was employed to evaluate the molecular motion behavior of 2TPE-NDTA and 2TPE2NDTA in the solid state. Single TPE was employed as control. The results manifested that the relaxation time of 2TPE-NDTA and 2TPE-2NDTA were largely decreased compared with that of single TPE, directly confirming the critical role of long alkyl chains in permitting the free intramolecular motion in the aggregated state. In order to evaluate whether the intramolecular rotation could generate enough heat, the photothermal conversion of 2TPENDTA NPs and 2TPE-2NDTA NPs utilizing DSPE$\mathrm{PEG}_{2000}$ as the encapsulating matrix was investigated. A high-performing semiconducting polymer, poly(cyclopentadithiophene-alt-benzothia-diazole), was also prepared into SPNs as the control. Encouragingly, 2TPENDTA NPs and 2TPE-2NDTA NPs both exhibited higher photothermal conversion efficiencies than SPNs, demonstrating the excellent heat generating ability of the intramolecular rotation. Specifically, 2TPE-2NDTA NPs showed the highest photothermal conversion efficiency among these three NPs. These results are consistent with the greatly reduced fluorescence quantum yield of 2TPENDTA NPs and 2TPE-2NDTA NPs compared with that of NDTA NPs, which facilitated the dominated nonradiative decay in the exciton relaxation process. Based on the above results, Tang, Ding and co-workers thus put forward a concept of "intramolecular motion-induced photothermy (iMIPT)" as a new molecular guideline to develop advanced photothermal agents. Contrary to the "aggregation-induced emission" (AIE) process, which suppresses active intramolecular motion to emit bright light in the aggregates [93-100], iMIPT process favors the active excited-state intramolecular motion to generate heat in aggregates (Fig. 9). Owing to the excellent pho- tothermal conversion efficiency and inherent EPR effect, 2TPE-2NDTA NPs exhibited an outstanding performance in the PA imaging of tumors in vivo with highcontrast. This study highlights a new mechanism based on iMIPT to develop advanced organic/polymer photothermal agents with high photothermal conversion efficiencies, which is believed to promote the application of PA imaging and PTT profoundly.

\section{Adjusting TICT in aggregates for boosting photothermal property}

Excited-state intramolecular motion was also found to induce the formation of twisted intramolecular charge transfer (TICT) state, which returned to the ground state mainly through thermal deactivation as well. Due to the susceptibility to environment, TICT thus has great potential to enhance the heat generation [101]. In addition, the long alkyl chain could also regulate the TICT properties of photothermal agents while facilitating the excited-state intramolecular motion. Therefore, it is speculated that regulating the TICT properties in the molecular motion state should be beneficial to the development of advanced photothermal agents with further elevated photothermal conversion efficiency.

In order to verify this speculation, Tang, Ding, Shi and co-workers [102] further developed four NIR-absorbing organic small molecule photothermal agents featured with a linear 1-hexyl unit, and three second-position branched alkyl groups including 2-ethylhexyl, 2-octyldecyl and 2-decylmyristyl, denoted as NIR6, NIRb6, NIRb10 and NIRb14, respectively (Fig. 10a). Due to the weak steric hindrance to induce the molecular motion in aggregates, NIR6 was designed to compare the effect of molecular motion with NIRb6. While NIRb6, NIRb10 and NIRb14 with suitable steric hindrance and increasing chain length were designed to evaluate the photothermal effect of TICT with different degrees, considering the increasing ICT rate constant with the extended length of the alkyl chains and the increasing dihedral angle between electron-withdrawing benzo[1,2-c:4,5-c' $]$ bis $([1,2,5]$ thiadiazole) (BBTD) and electron-donating thiophene from NIRb6 to NIRb14 (Fig. 10b). Photothermal experiments exhibited a higher photothermal conversion of NIRb6 NPs than that of NIR6 NPs, confirming the heat generation ability of molecular motion in aggregates. Moreover, the critical role of TICT to further enhance the heat generation was demonstrated by the gradually increasing photothermal conversion efficiencies of NIRb6, NIRb10 and NIRb14 NPs. Thus, Tang, Ding and co-workers put forward a concept of "adjusting TICT in aggregates for 

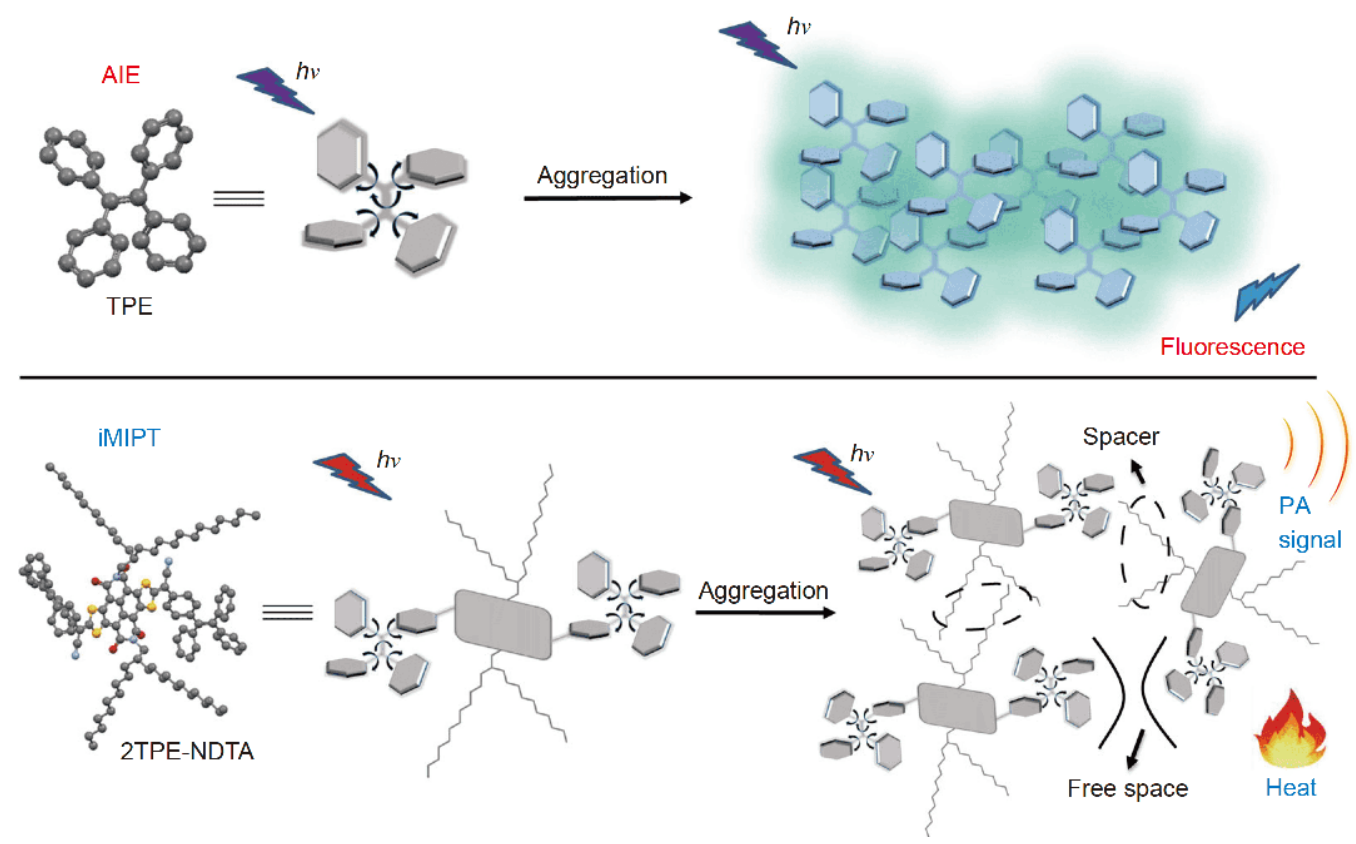

Figure 9 Schematic illustration of the working mechanisms of AIE and iMIPT using TPE and 2TPE-NDTA as the example, respectively. Reproduced with permission from Ref. [92]. Copyright 2019, Springer Nature.

boosting photothermal property" as a new mechanism to develop advanced photothermal agents. Afterwards, in order to apply NIRb14 to the diagnosis and treatment of tumors in vivo, $\operatorname{poly}(\beta$-amino ester)- $b$-poly(caprolactone) (PAE- $b$-PCL) and poly(ethylene glycol)-block-poly(caprolactone) (PEG- $b$-PCL) were introduced to afford tumor microenvironment-responsive NIRb14-PAE/PEG NPs. Thanks to the prolonged blood circulation time and enhanced tumor accumulation, as well as the high photothermal conversion efficiency, NIRb14-PAE/PEG NPs exhibited a superb PA imaging contrast and PTT efficacy in vivo of mice. This study highlights a new molecular motion mechanism, "adjusting TICT in aggregates for boosting photothermal property", to develop advanced organic/polymer photothermal agents with further elevated non-radiative decay efficiencies for enhanced PA imaging and PTT effect.

\section{CONCLUSIONS AND PERSPECTIVES}

Organic/polymer photothermal nanoagents including semiconducting polymer NPs and small-molecule organic photothermal agents-encapsulated NPs give excellent performance in in vivo PA imaging and PTT, due to their excellent optical properties, good biocompatibility, easy processability, and flexible surface functionalization. The in vivo PA and PTT efficiencies of the organic/polymer photothermal nanoagents are closely related to their ex- tinction coefficients, non-radiative decay efficiencies and Grüneisen coefficients, as well as the heat interfacial conductance from the nanoagents to water, the tissue penetrating ability of light absorbing and the amount of nanoagents at the targeted sites. This review summarizes the recent advances in the design strategies of organic/ polymer photothermal nanoagents for PA imaging and PTT applications. Specifically, molecular structure screening strategy is an important approach for enhanced PA signals by screening organic photothermal agents with high extinction coefficients. However, this strategy lacks theoretical guidance and consumes lots of time. To overcome this limitation, a general molecular structure engineering design guideline to enhance the PA imaging and PTT effect of organic photothermal agents is proposed by increasing their $\mathrm{D}-\mathrm{A}$ strength, which simultaneously aggrandizes their extinction coefficients and nonradiative decay efficiencies. Considering the difficult synthesis of molecular structure engineering strategy, nanoengineering approach is an alternative means to simply and effectively improve the PA imaging and PTT effect of organic/polymer photothermal nanoagents. Nano-engineering approach consists of intraparticle nanoengineering approach and surface nanoengineering approach, which increases the non-radiative decay efficiencies and the heat interfacial conductance from the nanoagents to water, respectively. Stimuli-controlled NP 


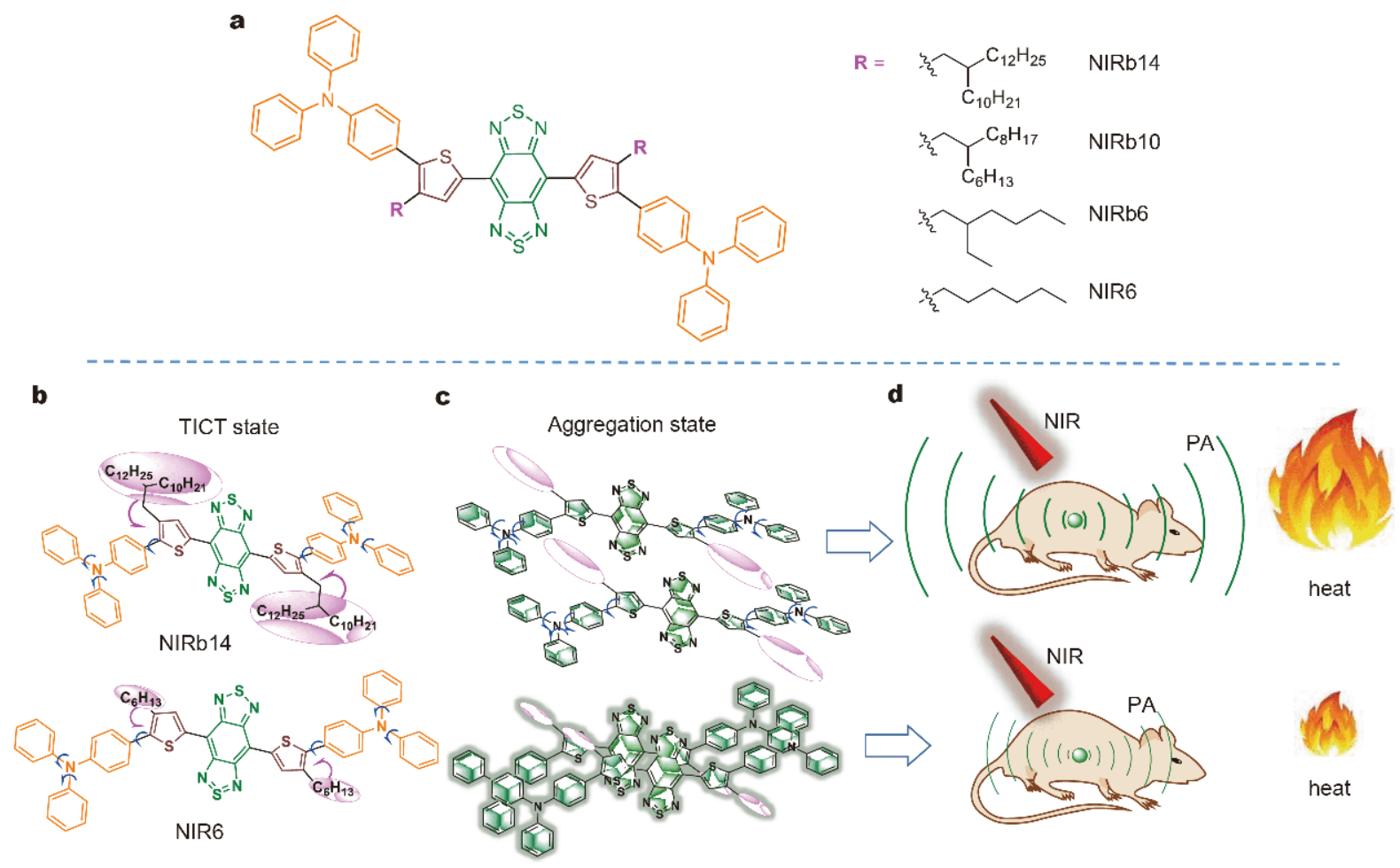

Figure 10 (a) Molecular structures of NIR6, NIRb6, NIRb10 and NIRb14. (b, c) Schematic diagram of the TITC states of NIR6 and NIRb14 in solution (b) and aggregation state (c). (d) Schematic diagram of the different PA imaging-guided PTT effects employing NIR6 NPs and NIRb14 NPs as the photothermal agents, respectively. Reproduced with permission from Ref. [102], Copyright 2019, American Chemical Society.

regrowth is another efficient approach to activate and enhance the PA imaging and PTT effect of organic/ polymer photothermal nanoagents, which increases the rate of heat transfer within NPs. Moreover, since photons need to penetrate tissues to supply energy to photothermal nanoagents during in vivo applications, extending the absorption wavelength to the NIR-II window, which increases the light energy harvested by photothermal nanoagents at deep tissues, is also an effective way to enhance the PA imaging and PTT effect of organic/polymer photothermal nanoagents. However, in the application of PA imaging and PTT of tumors in vivo, photothermal nanoagents are generally largely recognized by the immune system and mostly cleared by the liver and kidney as a result of their exogeneity, resulting in a relatively low nanoagents accumulation in the tumor sites and hence a non-ideal PA imaging and PTT effect. To resolve this issue, a general approach to enhance the PA imaging and PTT effect of tumors in vivo is proposed by designing biomimetic photothermal nanoagents with reduced accumulation in the liver and kidney and increased accumulation at the tumor sites. In addition, as an important class of organic/polymer photothermal na- noagents, small-molecule organic photothermal agentsencapsulated NPs generally suffer from the poor stability issue, which leads to subdued PA imaging and PTT effect. Thus, increasing the stability of small-molecule organic photothermal agents, which essentially maintain nearly constant extinction coefficients and photothermal conversion efficiencies in in vivo applications, is also a general approach to enhance the in vivo PA imaging and PTT effect of organic/polymer photothermal nanoagents. Besides, in some cases, organic/polymer photothermal nanoagents with dual-modality PA and fluorescence imaging ability are required, due to the complementarity of high spatial resolution of PA imaging and high sensitivity of fluorescence imaging. However, according to Jablonski diagram, fluorescence emission and thermal deactivation are two competitive dissipation pathways of the light excitation energy absorbed by chromophores, which implies that fluorescence and PA imaging of one single optical material could not be maximized at the same time. To address this dilemma, a function-transformable strategy based on molecular structural transformations was proposed to maximize the PA and fluorescence imaging efficiencies of optical agents on 
demand. At last, two new emerging mechanisms based on "intramolecular motion-induced photothermy" and "adjusting TICT in aggregates for boosting photothermal property" are highlighted to develop advanced organic photothermal agents with high photothermal conversion efficiencies, which are believed to promote the application of PA imaging and PTT profoundly.

Although great achievements have been made in the development of advanced organic/polymer photothermal nanoagents following these above strategies, many challenges still exist. Despite of the nontoxicity exhibited by cell and mouse experiments, the long-term toxicity of organic/polymer photothermal nanoagents, especially SPNs, has not been fully understood. Designing SPNs with biodegradation ability or ultrasmall sizes $(<5 \mathrm{~nm})$, which endow SPNs with fast renal clearance capability, is a practicable approach to resolve this issue. In addition, as most of the current studies have achieved enhanced PA imaging and PTT effects of organic/polymer photothermal nanoagents by adjusting their extinction coefficients and non-radiative decay efficiencies, advanced nanoagents based on the regulation of Grüneisen coefficients are still rare. Large amount of researches on regulating Grüneisen coefficients are required to be conducted to fully elucidate its mechanism. Moreover, compared with fluorescence imaging, the detection sensitivity of PA imaging still has a large room to be optimized. Integration of traditional approaches and new emerging mechanisms, e.g., developing NIR-II light-absorbing iMIPT luminogens or conjugated polymers with iMIPT characteristic, may be an effective strategy to improve the detection sensitivity of PA imaging, which could in turn conduce to the reduction of toxicity by lowering the dosage of photothermal nanoagents administrated. Considering more and more attention attracted by the excellent PA imaging and PTT capabilities of organic/polymer photothermal nanoagents, these above issues are expected to be resolved in the near future, and advanced organic/polymer photothermal nanoagents with adaptive photothermal function and efficacy obtained under the guidance of photophysical property regulation will finally benefit the advancement of disease theranostics.

Received 15 May 2019; accepted 1 July 2019; published online 6 August 2019

1 Kim JW, Galanzha EI, Shashkov EV, et al. Golden carbon nanotubes as multimodal photoacoustic and photothermal highcontrast molecular agents. Nat Nanotech, 2009, 4: 688-694

2 Liu Y, Bhattarai P, Dai Z, et al. Photothermal therapy and pho- toacoustic imaging via nanotheranostics in fighting cancer. Chem Soc Rev, 2019, 48: 2053-2108

3 Jokerst JV, Gambhir SS. Molecular imaging with theranostic nanoparticles. Acc Chem Res, 2011, 44: 1050-1060

4 Ke H, Wang J, Dai Z, et al. Gold-nanoshelled microcapsules: a theranostic agent for ultrasound contrast imaging and photothermal therapy. Angew Chem Int Ed, 2011, 50: 3017-3021

5 Zhang D, Qi GB, Zhao YX, et al. In situ formation of nanofibers from purpurin18-peptide conjugates and the assembly induced retention effect in tumor sites. Adv Mater, 2015, 27: 6125-6130

6 Lyu Y, Zeng J, Jiang Y, et al. Enhancing both biodegradability and efficacy of semiconducting polymer nanoparticles for photoacoustic imaging and photothermal therapy. ACS Nano, 2018, 12: $1801-1810$

7 Mou J, Chen Y, Ma M, et al. Facile synthesis of liposome/ $/ \mathrm{Cu}_{2-x} \mathrm{~S}-$ based nanocomposite for multimodal imaging and photothermal therapy. Sci China Mater, 2015, 58: 294-301

8 Kim C, Favazza C, Wang LV. In vivo photoacoustic tomography of chemicals: high-resolution functional and molecular optical imaging at new depths. Chem Rev, 2010, 110: 2756-2782

9 Zhang D, Wang Z, Wang L, et al. High-performance identification of human bladder cancer using a signal self-amplifiable photoacoustic nanoprobe. ACS Appl Mater Interfaces, 2018, 10: 28331-28339

10 Liu Y, Teng L, Liu HW, et al. Recent advances in organic-dyebased photoacoustic probes for biosensing and bioimaging. Sci China Chem, 2019, 24: doi: 10.1007/s11426-019-9506-2

11 Nie L, Chen X. Structural and functional photoacoustic molecular tomography aided by emerging contrast agents. Chem Soc Rev, 2014, 43: 7132-7170

12 Wang $\mathrm{LV}, \mathrm{Hu}$ S. Photoacoustic tomography: in vivo imaging from organelles to organs. Science, 2012, 335: 1458-1462

13 Zhang HF, Maslov K, Stoica G, et al. Functional photoacoustic microscopy for high-resolution and noninvasive in vivo imaging. Nat Biotechnol, 2006, 24: 848-851

14 Jung HS, Verwilst P, Sharma A, et al. Organic molecule-based photothermal agents: an expanding photothermal therapy universe. Chem Soc Rev, 2018, 47: 2280-2297

15 Yang K, Zhang S, Zhang G, et al. Graphene in mice: ultrahigh in vivo tumor uptake and efficient photothermal therapy. Nano Lett, 2010, 10: 3318-3323

16 Li Y, Bai X, Xu M, et al. Photothermo-responsive $\mathrm{Cu}_{7} \mathrm{~S}_{4} @$ polymer nanocarriers with small sizes and high efficiency for controlled chemo/photothermo therapy. Sci China Mater, 2016, 59: 254-264

17 Gao J, Sanchez-Purra M, Huang H, et al. Synthesis of differentsized gold nanostars for Raman bioimaging and photothermal therapy in cancer nanotheranostics. Sci China Chem, 2017, 60: 1219-1229

18 Qi J, Fang Y, Kwok RTK, et al. Highly stable organic small molecular nanoparticles as an advanced and biocompatible phototheranostic agent of tumor in living mice. ACS Nano, 2017, 11: $7177-7188$

19 Zhang J, Yang C, Zhang R, et al. Biocompatible D-A semiconducting polymer nanoparticle with light-harvesting unit for highly effective photoacoustic imaging guided photothermal therapy. Adv Funct Mater, 2017, 27: 1605094

20 Chen D, Wang C, Nie X, et al. Photoacoustic imaging guided near-infrared photothermal therapy using highly water-dispersible single-walled carbon nanohorns as theranostic agents. Adv Funct Mater, 2014, 24: 6621-6628 
21 Mou J, Li P, Liu C, et al. Ultrasmall $\mathrm{Cu}_{2-x} \mathrm{~S}$ nanodots for highly efficient photoacoustic imaging-guided photothermal therapy. Small, 2015, 11: 2275-2283

$22 \mathrm{Ni} \mathrm{D}$, Jiang $\mathrm{D}$, Valdovinos $\mathrm{HF}$, et al. Bioresponsive polyoxometalate cluster for redox-activated photoacoustic imagingguided photothermal cancer therapy. Nano Lett, 2017, 17: 32823289

23 Mallidi S, Luke GP, Emelianov S. Photoacoustic imaging in cancer detection, diagnosis, and treatment guidance. Trends Biotech, 2011, 29: 213-221

24 Yang Z, Song J, Dai Y, et al. Self-assembly of semiconductingplasmonic gold nanoparticles with enhanced optical property for photoacoustic imaging and photothermal therapy. Theranostics, 2017, 7: 2177-2185

25 Zheng X, Mao H, Huo D, et al. Successively activatable ultrasensitive probe for imaging tumour acidity and hypoxia. Nat Biomed Eng, 2017, 1: 0057

26 Chen Y, Shi J. Mesoporous carbon biomaterials. Sci China Mater, 2015, 58: 241-257

27 Gao YJ, Qiao ZY, Wang H. Polymers with tertiary amine groups for drug delivery and bioimaging. Sci China Chem, 2016, 59: 9911002

28 Huang $\mathrm{P}$, Lin J, Li W, et al. Biodegradable gold nanovesicles with an ultrastrong plasmonic coupling effect for photoacoustic imaging and photothermal therapy. Angew Chem Int Ed, 2013, 52: 13958-13964

29 Yang T, Tang Y, Liu L, et al. Size-dependent $\mathrm{Ag}_{2} \mathrm{~S}$ nanodots for second near-infrared fluorescence/photoacoustics imaging and simultaneous photothermal therapy. ACS Nano, 2017, 11: 18481857

30 Zhang S, Guo W, Wei J, et al. Terrylenediimide-based intrinsic theranostic nanomedicines with high photothermal conversion efficiency for photoacoustic imaging-guided cancer therapy. ACS Nano, 2017, 11: 3797-3805

31 Zhu H, Lai Z, Fang Y, et al. Ternary chalcogenide nanosheets with ultrahigh photothermal conversion efficiency for photoacoustic theranostics. Small, 2017, 13: 1604139

32 Shashkov EV, Everts M, Galanzha EI, et al. Quantum dots as multimodal photoacoustic and photothermal contrast agents. Nano Lett, 2008, 8: 3953-3958

33 Borg RE, Rochford J. Molecular photoacoustic contrast agents: design principles \& applications. Photochem Photobiol, 2018, 94: 1175-1209

34 Frenette M, Hatamimoslehabadi M, Bellinger-Buckley S, et al. Shining light on the dark side of imaging: excited state absorption enhancement of a bis-styryl BODIPY photoacoustic contrast agent. J Am Chem Soc, 2014, 136: 15853-15856

35 Lyu Y, Fang Y, Miao Q, et al. Intraparticle molecular orbital engineering of semiconducting polymer nanoparticles as amplified theranostics for in vivo photoacoustic imaging and photothermal therapy. ACS Nano, 2016, 10: 4472-4481

36 Aoki H, Nojiri M, Mukai R, et al. Near-infrared absorbing polymer nano-particle as a sensitive contrast agent for photoacoustic imaging. Nanoscale, 2015, 7: 337-343

37 Yang $\mathrm{K}, \mathrm{Xu} \mathrm{H}$, Cheng $\mathrm{L}$, et al. In vitro and in vivo near-infrared photothermal therapy of cancer using polypyrrole organic nanoparticles. Adv Mater, 2012, 24: 5586-5592

38 Hong G, Antaris AL, Dai H. Near-infrared fluorophores for biomedical imaging. Nat Biomed Eng, 2017, 1: 0010

39 Ding F, Zhan Y, Lu X, et al. Recent advances in near-infrared II fluorophores for multifunctional biomedical imaging. Chem Sci, 2018, 9: 4370-4380

40 Song G, Liang C, Gong $\mathrm{H}$, et al. Core-shell $\mathrm{MnSe} @ \mathrm{Bi}_{2} \mathrm{Se}_{3}$ fabricated via a cation exchange method as novel nanotheranostics for multimodal imaging and synergistic thermoradiotherapy. Adv Mater, 2015, 27: 6110-6117

41 Chen S, Jia Q, Zheng X, et al. PEGylated carbon $\operatorname{dot} / \mathrm{MnO}_{2}$ nanohybrid: a new $\mathrm{pH} / \mathrm{H}_{2} \mathrm{O}_{2}$-driven, turn-on cancer nanotheranostics. Sci China Mater, 2018, 61: 1325-1338

42 Huang $\mathrm{P}$, Lin J, Wang X, et al. Light-triggered theranostics based on photosensitizer-conjugated carbon dots for simultaneous enhanced-fluorescence imaging and photodynamic therapy. Adv Mater, 2012, 24: 5104-5110

43 Kim J, Park S, Lee C, et al. Organic nanostructures for photoacoustic imaging. ChemNanoMat, 2016, 2: 156-166

44 Geng J, Sun C, Liu J, et al. Biocompatible conjugated polymer nanoparticles for efficient photothermal tumor therapy. Small, 2015, 11: 1603-1610

45 Xiong Z, Shen M, Shi X. Dendrimer-based strategies for cancer therapy: recent advances and future perspectives. Sci China Mater, 2018, 61: 1387-1403

46 Li J, Pu K. Development of organic semiconducting materials for deep-tissue optical imaging, phototherapy and photoactivation. Chem Soc Rev, 2019, 48: 38-71

47 Li K, Liu B. Polymer-encapsulated organic nanoparticles for fluorescence and photoacoustic imaging. Chem Soc Rev, 2014, 43: 6570-6597

48 Weber J, Beard PC, Bohndiek SE. Contrast agents for molecular photoacoustic imaging. Nat Methods, 2016, 13: 639-650

49 Chen P, Ma Y, Zheng Z, et al. Facile syntheses of conjugated polymers for photothermal tumour therapy. Nat Commun, 2019, 10: 1192

50 Jiang $\mathrm{Y}, \mathrm{Pu}$ K. Multimodal biophotonics of semiconducting polymer nanoparticles. Acc Chem Res, 2018, 51: 1840-1849

$51 \mathrm{Pu} \mathrm{K}$, Shuhendler AJ, Jokerst JV, et al. Semiconducting polymer nanoparticles as photoacoustic molecular imaging probes in living mice. Nat Nanotech, 2014, 9: 233-239

52 Zhang X, Xia LY, Chen X, et al. Hydrogel-based phototherapy for fighting cancer and bacterial infection. Sci China Mater, 2017, 60: 487-503

53 Gunnlaugsson T. Integrating NIR light photocage enables in vivo dual-channel tracking of drug release. Sci China Chem, 2018, 61: 1347-1348

54 Szabó C, Ischiropoulos H, Radi R. Peroxynitrite: biochemistry, pathophysiology and development of therapeutics. Nat Rev Drug Discov, 2007, 6: 662-680

55 Medzhitov R. Origin and physiological roles of inflammation. Nature, 2008, 454: 428-435

56 Guo B, Sheng Z, Hu D, et al. Molecular engineering of conjugated polymers for biocompatible organic nanoparticles with highly efficient photoacoustic and photothermal performance in cancer theranostics. ACS Nano, 2017, 11: 10124-10134

$57 \mathrm{Ng} \mathrm{KK}$, Zheng G. Molecular interactions in organic nanoparticles for phototheranostic applications. Chem Rev, 2015, 115: 1101211042

58 Lovell JF, Jin CS, Huynh E, et al. Porphysome nanovesicles generated by porphyrin bilayers for use as multimodal biophotonic contrast agents. Nat Mater, 2011, 10: 324-332

59 Chen $\mathrm{C}, \mathrm{Ou} \mathrm{H}$, Liu R, et al. Regulating the photophysical property of organic/polymer optical agents for promoted cancer photo- 
theranostics. Adv Mater, 2019, 35: 1806331

60 Cai X, Liu J, Liew WH, et al. Organic molecules with propeller structures for efficient photoacoustic imaging and photothermal ablation of cancer cells. Mater Chem Front, 2017, 1: 1556-1562

61 Dong T, Wen K, Chen J, et al. Significant enhancement of photothermal and photoacoustic efficiencies for semiconducting polymer nanoparticles through simply molecular engineering. Adv Funct Mater, 2018, 28: 1800135

62 Miao Q, Lyu Y, Ding D, et al. Semiconducting oligomer nanoparticles as an activatable photoacoustic probe with amplified brightness for in vivo imaging of $\mathrm{pH}$. Adv Mater, 2016, 28: 36623668

63 Coakley RJ, Taggart C, McElvaney NG, et al. Cytosolic pH and the inflammatory microenvironment modulate cell death in human neutrophils after phagocytosis. Blood, 2002, 100: 3383-3391

64 Webb BA, Chimenti M, Jacobson MP, et al. Dysregulated pH: a perfect storm for cancer progression. Nat Rev Cancer, 2011, 11: 671-677

65 Zhen X, Feng X, Xie C, et al. Surface engineering of semiconducting polymer nanoparticles for amplified photoacoustic imaging. Biomaterials, 2017, 127: 97-106

66 Xie C, Zhen X, Lyu Y, et al. Nanoparticle regrowth enhances photoacoustic signals of semiconducting macromolecular probe for in vivo imaging. Adv Mater, 2017, 29: 1703693

67 Li LL, Ma HL, Qi GB, et al. Pathological-condition-driven construction of supramolecular nanoassemblies for bacterial infection detection. Adv Mater, 2016, 28: 254-262

68 Yang Z, Tian R, Wu J, et al. Impact of semiconducting perylene diimide nanoparticle size on lymph node mapping and cancer imaging. ACS Nano, 2017, 11: 4247-4255

69 Guo B, Sheng Z, Hu D, et al. Through scalp and skull NIR-II photothermal therapy of deep orthotopic brain tumors with precise photoacoustic imaging guidance. Adv Mater, 2018, 30: 1802591

70 Yu S, Tu D, Lian W, et al. Lanthanide-doped near-infrared II luminescent nanoprobes for bioapplications. Sci China Mater, 2019, 62: 1071-1086

71 Antaris AL, Chen $\mathrm{H}$, Cheng $\mathrm{K}$, et al. A small-molecule dye for NIR-II imaging. Nat Mater, 2016, 15: 235-242

$72 \mathrm{Lu} \mathrm{W}$, Melancon MP, Xiong C, et al. Effects of photoacoustic imaging and photothermal ablation therapy mediated by targeted hollow gold nanospheres in an orthotopic mouse xenograft model of glioma. Cancer Res, 2011, 71: 6116-6121

73 Lee C, Hwang HS, Lee S, et al. Rabies virus-inspired silica-coated gold nanorods as a photothermal therapeutic platform for treating brain tumors. Adv Mater, 2017, 29: 1605563

74 Zheng X, Wang X, Mao H, et al. Hypoxia-specific ultrasensitive detection of tumours and cancer cells in vivo. Nat Commun, 2015, 6: 5834

75 Li J, Liu J, Chen C. Remote control and modulation of cellular events by plasmonic gold nanoparticles: implications and opportunities for biomedical applications. ACS Nano, 2017, 11: 2403-2409

76 Zhang J, Chen J, Ren J, et al. Biocompatible semiconducting polymer nanoparticles as robust photoacoustic and photothermal agents revealing the effects of chemical structure on high photothermal conversion efficiency. Biomaterials, 2018, 181: 92-102

77 Torchilin V. Tumor delivery of macromolecular drugs based on the EPR effect. Adv Drug Deliver Rev, 2011, 63: 131-135

78 McPherson T, Kidane A, Szleifer I, et al. Prevention of protein adsorption by tethered poly(ethylene oxide) layers: Experiments and single-chain mean-field analysis. Langmuir, 1998, 14: 176186

79 Zhao J, Chai YD, Zhang J, et al. Long circulating micelles of an amphiphilic random copolymer bearing cell outer membrane phosphorylcholine zwitterions. Acta Biomater, 2015, 16: 94-102

80 Knop K, Hoogenboom R, Fischer D, et al. Poly(ethylene glycol) in drug delivery: Pros and cons as well as potential alternatives. Angew Chem Int Ed, 2010, 49: 6288-6308

81 Owens III D, Peppas N. Opsonization, biodistribution, and pharmacokinetics of polymeric nanoparticles. Int J Pharm, 2006, 307: 93-102

82 Chai Z, Hu X, Lu W. Cell membrane-coated nanoparticles for tumor-targeted drug delivery. Sci China Mater, 2017, 60: 504-510

83 Chen Z, Zhao P, Luo Z, et al. Cancer cell membrane-biomimetic nanoparticles for homologous-targeting dual-modal imaging and photothermal therapy. ACS Nano, 2016, 10: 10049-10057

84 Feng G, Liu B. Multifunctional AIEgens for future theranostics. Small, 2016, 12: 6528-6535

85 Yang K, Hu L, Ma X, et al. Multimodal imaging guided photothermal therapy using functionalized graphene nanosheets anchored with magnetic nanoparticles. Adv Mater, 2012, 24: 18681872

86 Huang $\mathrm{P}$, Rong $\mathrm{P}$, Jin A, et al. Dye-loaded ferritin nanocages for multimodal imaging and photothermal therapy. Adv Mater, 2014, 26: 6401-6408

87 Qi J, Chen C, Zhang X, et al. Light-driven transformable optical agent with adaptive functions for boosting cancer surgery outcomes. Nat Commun, 2018, 9: 1848

88 Lv JA, Liu Y, Wei J, et al. Photocontrol of fluid slugs in liquid crystal polymer microactuators. Nature, 2016, 537: 179-184

89 Qian H, Cousins ME, Horak EH, et al. Suppression of Kasha's rule as a mechanism for fluorescent molecular rotors and aggregation-induced emission. Nat Chem, 2017, 9: 83-87

90 Goh WL, Lee MY, Joseph TL, et al. Molecular rotors as conditionally fluorescent labels for rapid detection of biomolecular interactions. J Am Chem Soc, 2014, 136: 6159-6162

91 Jin M, Chung TS, Seki T, et al. Phosphorescence control mediated by molecular rotation and aurophilic interactions in amphidynamic crystals of 1,4-bis[tri-( $p$-fluorophenyl)phosphane-gold(I)ethynyl]benzene. J Am Chem Soc, 2017, 139: 18115-18121

92 Zhao Z, Chen $\mathrm{C}$, Wu W, et al. Highly efficient photothermal nanoagent achieved by harvesting energy via excited-state intramolecular motion within nanoparticles. Nat Commun, 2019, 10: 768

93 Ni X, Zhang X, Duan X, et al. Near-infrared afterglow luminescent aggregation-induced emission dots with ultrahigh tumor-toliver signal ratio for promoted image-guided cancer surgery. Nano Lett, 2019, 19: 318-330

94 Mei J, Leung NLC, Kwok RTK, et al. Aggregation-induced emission: Together we shine, united we soar! Chem Rev, 2015, 115: $11718-11940$

95 Gao H, Zhang X, Chen C, et al. Unity makes strength: How aggregation-induced emission luminogens advance the biomedical field. Adv Biosys, 2018, 2: 1800074

96 Hong Y, Lam JWY, Tang BZ. Aggregation-induced emission: phenomenon, mechanism and applications. Chem Commun, 2009, 1: 4332-4353

97 Liang J, Tang BZ, Liu B. Specific light-up bioprobes based on AIEgen conjugates. Chem Soc Rev, 2015, 44: 2798-2811 
98 Jiang N, Shen T, Sun JZ, et al. Aggregation-induced emission: Right there shining. Sci China Mater, 2019, 62: 1227-1235

99 Ou H, Dai S, Liu R, et al. Manipulating the intramolecular motion of AIEgens for boosted biomedical applications. Sci China Chem, 2019, 62: 929-932

$100 \mathrm{Xu} \mathrm{M}$, Wang X, Wang Q, et al. Analyte-responsive fluorescent probes with AIE characteristic based on the change of covalent bond. Sci China Mater, 2019, 62: 1236-1250

101 Grabowski ZR, Rotkiewicz K, Rettig W. Structural changes accompanying intramolecular electron transfer: focus on twisted intramolecular charge-transfer states and structures. Chem Rev, 2003, 103: 3899-4032

102 Liu S, Zhou X, Zhang H, et al. Molecular motion in aggregates: manipulating TICT for boosting photothermal theranostics. J Am Chem Soc, 2019, 141: 5359-5368

Acknowledgements This work was supported by the National Natural Science Foundation of China (51622305, 51873092, 31771031, and 81701829), the National Basic Research Program of China (2015CB856503), the National Key Research and Development Program of China (2018YFA0209800), and the Fundamental Research Funds for the Central Universities, Nankai University (63191521, 63171218 and 63191176).

Author contributions All the authors contributed to the discussion and writing of the manuscript.

Conflict of interest The authors declare that they have no conflict of interest.

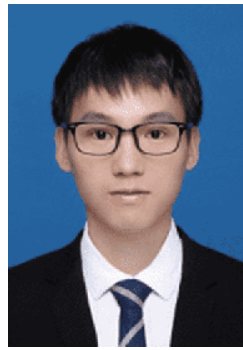

Hanlin Ou received his $\mathrm{PhD}$ degree from the College of Chemistry in Nankai University in 2018. Afterwards he joined the College of Life Sciences in Nankai University to conduct his postdoctoral research under the supervision of Prof. Dan Ding. His current research focuses on the biomedical applications of functional molecular imaging probes.

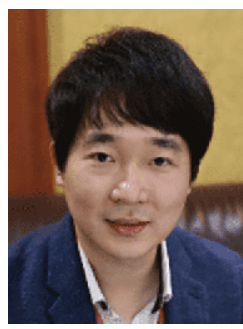

Dan Ding received his $\mathrm{PhD}$ degree from the Department of Polymer Science and Engineering in Nanjing University in 2010. After a postdoctoral training in National University of Singapore, he joined Nankai University, where he is currently a professor in the State Key Laboratory of Medicinal Chemical Biology, Key Laboratory of Bioactive Materials, Ministry of Education, and College of Life Sciences. He also conducted his work in The Hong Kong University of Science and Technology as a visiting scholar. His current research focuses on the design and synthesis of smart/functional molecular imaging probes and exploration of their biomedical applications.

\section{有机高分子纳米光热剂在体内疾病诊疗中的应用}

欧翰林 ${ }^{1}$, 李军 ${ }^{1}$, 陈超 ${ }^{1}$, 高贺麒 ${ }^{1}$, 薛雪 ${ }^{2^{*}}$, 丁 丹 $^{1,2,3^{*}}$

摘要 有机高分子纳米光热剂具有生物安全性高、易于功能化修 饰和诊疗一体化等优点, 因而被广泛应用于生物医学领域. 本综述 主要总结了近年来有机高分子纳米光热剂在体内光声成像和光热 治疗中的应用进展, 并重点讨论了通过调控有机高分子纳米光热 剂的光物理性质以实现增强的体内光声成像和光热治疗效果的传 统策略和新兴机理. 希望本综述为开发更多具有优异体内光声成 像和光热治疗效果的有机高分子纳米光热剂提供借鉴, 以进一步 推动肿瘤等重大疾病的早期诊断和治疗. 